\title{
Possible interpretations of IceCube high-energy neutrino events
}

\author{
Chee Sheng Fong, ${ }^{a}$ Hisakazu Minakata, ${ }^{a, b}$ Boris Panes ${ }^{a}$ \\ and Renata Zukanovich Funchal ${ }^{a, b}$ \\ ${ }^{a}$ Instituto de Fúsica, Universidade de São Paulo, \\ C.P. 66.318, 05315-970 São Paulo, Brazil \\ ${ }^{b}$ Kavli Institute for Theoretical Physics, University of California, \\ Santa Barbara, CA 93106-4030, U.S.A. \\ E-mail: fong@if.usp.br, minakata@fmail.if.usp.br, bapanes@if.usp.br, \\ zukanov@if.usp.br
}

ABSTRACT: We discuss possible interpretations of the 37 high energy neutrino events observed by the IceCube experiment in the South Pole. We examine the possibility to explain the observed neutrino spectrum exclusively by the decays of a heavy long-lived particle of mass in the $\mathrm{PeV}$ range. We compare this with the standard scenario, namely, a single power-law spectrum related to neutrinos produced by astrophysical sources and a viable hybrid situation where the spectrum is a product of two components: a power-law and the long-lived particle decays. We present a simple extension of the Standard Model that could account for the heavy particle decays that are needed in order to explain the data. We show that the current data equally supports all above scenarios and try to evaluate the exposure needed in order to falsify them in the future.

KeYwords: Cosmology of Theories beyond the SM, Solar and Atmospheric Neutrinos

ARXIV EPRINT: 1411.5318 


\section{Contents}

1 Introduction 1

2 Can a decaying Long-Lived Particle alone explain the IceCube data? 3

3 IceCube data analysis: a brief description 4

4 Fitting the IceCube data in three scenarios $\quad 6$

4.1 Power-law fit $\left(\mathrm{H}_{0}=\mathrm{I}\right) \quad 6$

4.2 Power-law + Long-Lived Particle two body decay fit $\left(\mathrm{H}_{0}=\mathrm{II}\right) \quad 8$

4.3 Pure Long-Lived Particle decays fit $\left(\mathrm{H}_{0}=\mathrm{III}, \mathrm{IV}\right.$ and V) 8

4.4 Constraints from gamma-ray and antiproton observations 11

5 Future data perspectives $\quad 12$

6 A model for a Long-Lived Particle 13

$\begin{array}{lll}6.1 \text { The scalar sector } & 14\end{array}$

$\begin{array}{ll}\text { 6.2 The fermionic sector } & 17\end{array}$

6.3 Does the Long-Lived Particle constitute most of the DM? 18

$\begin{array}{lll}7 \text { Conclusion } & 21\end{array}$

A Some details on the statistical treatment $\quad 22$

$\begin{array}{lll}\text { A.1 p-value } & 23\end{array}$

A.2 Intervals 23

A.3 Exclusion time 24

B Confidence intervals $\quad 25$

\section{Introduction}

The particle physics community around the globe invested a few decades in a variety of experiments in a coordinated effort to scrutinize the Standard Model (SM) of electroweak interactions. The scientific achievements turned out to be impressive. Neutrino flavour oscillations have been discovered and confirmed by a string of solar, atmospheric, reactor and accelerator neutrino experiments [1-7], challenging our understanding of neutrino properties and their role in nature. The discovery of the Higgs boson by the LHC experiments in $2012[8,9]$, finally set the crowning glory of the SM electroweak symmetry breaking mechanism to experimental probing. The IceCube neutrino observatory, located in the South Pole, reported in 201328 neutrino candidates in the energy range from 50 to $2000 \mathrm{TeV}$, 
constituting at the time a $4.1 \sigma$ excess over the expected atmospheric background [10]. This exciting discovery of high-energy cosmic neutrinos heralds the dawn of neutrino astronomy and drives intriguing questions. Where do these neutrinos come from? How were they produced? Can they shed a light on the long lasting quest for the origin of cosmic rays? Are they related to the elusive Dark Matter (DM)?

Since this first announcement many authors have investigated the possible origin of these events. Among the possibilities explored are viable astrophysical sources [11-21], a possible DM connection [22-27], a leptoquark resonance [28], the decay of massive neutrinos $[29,30]$, the decay of a very heavy long-lived particle [31, 32] or even the possibility that these events could be understood in terms of novel interactions that neutrinos have with the cosmic neutrino background [33-35]. Finally, in ref. [36] the authors showed that assuming a simple isotropic astrophysical power-law spectrum for the neutrinos, the current data is consistent with neutrino having only the SM interactions. ${ }^{1}$

Recently IceCube has updated their analysis to include three years of data for a live time of 988 days. They have now 37 neutrino events with energy from $30 \mathrm{TeV}$ to $2 \mathrm{PeV}$ [38], strongly disfavouring a purely atmospheric explanation at $5.7 \sigma$. Interestingly enough, there are still no events in the energy range from $\sim 400 \mathrm{TeV}$ to $1 \mathrm{PeV}$. This may simply be a statistical fluctuation or a signature from the underlying physics. Furthermore, the energy distribution indicates that there may be a cutoff in the spectrum at $\mathrm{PeV}$ energies.

In this paper we discuss interpretations of the IceCube high-energy excess events. Among the candidates so far examined the following two scenarios seem to be "standard" and relatively model-independent: (a) a power-law spectrum to which astrophysical sources are kept in mind, (b) decays of a heavy Long-Lived Particle (LLP), but with a background power-law spectrum component. We investigate here, in addition to these two, the possibility that (c) decays of a heavy LLP alone can explain the IceCube data in its entire energy region. The last possibility was also studied in refs. [23, 24]. We emphasize here that the LLP we are considering can generically constitute only a fraction of the total DM. With the current IceCube 3-year data [38] and using only energy spectrum information, we will show that all the three scenarios above (a)-(c) can fit the current data well. Although the analysis of the angular distribution of the events showed a preference for a DM-like distribution over the isotropic distribution, more data is required to draw a robust conclusion $[23,27]$. Hence in this work, we will only focus on energy spectrum information. The next question, then, is how these three scenarios can be distinguished. We investigate this problem by examining simulated data of IceCube for the future to establish the necessary exposure time to differentiate the three scenarios above.

This paper is organized as follows. In section 2, we discuss the generic properties of the LLP that are required to explain the features of the IceCube events. In section 3, we describe the various sources of neutrinos in IceCube required for our data analysis. In section 4, we perform an analysis of the IceCube data in view of the three scenarios (a)-(c). In section 5 we discuss how our model can be falsified with future IceCube data. In section 6 we construct a LLP model that could accommodate the IceCube observations.

\footnotetext{
${ }^{1}$ In ref. [37], the authors extended the study to consider two-component astrophysical flux.
} 
We discuss the LLP abundance in our model and conclude that it does not have to be a dominant part of the DM content of the Universe. Finally, in section 7 we make our final remarks and conclusions. This paper is completed with two appendices: in appendix A, we give additional details on the statistical treatment we employed in this work while in appendix B, we list the confidence intervals of the best fit parameters for various scenarios considered in this study.

\section{Can a decaying Long-Lived Particle alone explain the IceCube data?}

The spectacular detection of 37 neutrino events in the energy range from $30 \mathrm{TeV}$ to $2 \mathrm{PeV}$ within the three years data set of IceCube disfavours, as already mentioned, a purely atmospheric explanation at $5.7 \sigma$ [38]. Moreover, neutrino events are absent in the energy range from about $400 \mathrm{TeV}$ to $1 \mathrm{PeV}$ and beyond $2 \mathrm{PeV}$, while the assumption of an unbroken $E^{-2}$ flux, would predict three events beyond $2 \mathrm{PeV}$ [38]. So the non-observation of events at higher energies seem to suggest there is a cutoff at the PeV scale. Although at the moment this is still consistent with statistical fluctuations, here we opt for the exciting possibility that these features could arise from the decays of a single LLP. Clearly, more than one species of decaying LLP with different masses could as well describe these features. We will take the minimal point of view and stick to a single species of LLP in this work.

To accommodate the two features, the dip and the cutoff, described above, we need a decaying LLP, $Y$, with mass, $M_{Y}$, at the PeV scale and lifetime, $\tau_{Y}$, with the following properties:

(i) It has to be long-lived. ${ }^{2}$ In order for $Y$ to remain today, it has to have a lifetime at least longer than the lifetime of the Universe, i.e, $\tau_{Y}>t_{0} \simeq 4.4 \times 10^{17} \mathrm{s.}^{3}$

(ii) It has a two-body decay to at least a SM neutrino in the final state. Assuming the LLP to be non-relativistic, the two-body decay $Y \rightarrow \nu_{\alpha} N$ with $\nu_{\alpha}$ a SM neutrino of flavour $\alpha=e, \mu, \tau$ and $N$ can be neutrino or another SM singlet field, will produce a peak in the energy spectrum at $M_{Y} / 2$. The cutoff in the energy spectrum will be at $M_{Y} / 2$ as well. As shown in ref. [24], electroweak (EW) corrections can in fact soften the peak and give a low energy tail. Intriguingly, they also showed that a peak structure can also result from the decay $Y \rightarrow \bar{e} e$ due EW corrections (e.g. cascade radiation of massive gauge bosons) [40,41]. In the current work, we take into account $\mathrm{EW}$ corrections in a simple way by extrapolating the results of [41] to higher masses. We know this is not strictly correct as the EW corrections in that paper were computed at leading order and so their results are only valid as long as the particle is not too heavy. However, the calculation of higher order corrections would be beyond the scope of this work.

\footnotetext{
${ }^{2}$ This is probably obvious from its name.

${ }^{3}$ See, however, refs. [31, 32] where they discussed a scenario where the decaying particle can have a lifetime shorter than the age of the Universe but its mass has to be very heavy, around $\sim 10^{4} \mathrm{PeV}$.
} 
(iii) It has to admit at least another longer decay chain to neutrinos. In order to produce neutrinos with a continuum energy spectrum at lower energies, the LLP requires a longer decay chain to neutrinos, $Y \rightarrow \ldots \rightarrow \nu_{\alpha} \nu_{\beta} \ldots$, as considered, for instance, in refs. [23, 24, 42]. For example, if $Y$ is a scalar, we can have $Y \rightarrow \mu \bar{\mu}, Y \rightarrow \tau \bar{\tau}$, $Y \rightarrow t \bar{t}, Y \rightarrow 2 h$ etc. (we use the standard notation where $h$ is the SM Higgs, $t$ for top and so on). Using PYTHIA [43], we generated neutrino energy spectra from the decay of the LLP at rest with its mass at the PeV scale. Experimenting with various channels, we found that in order to account for the excess of neutrino events at lower energies (where the atmospheric neutrinos alone cannot explain the data), two-body decays such as $Y \rightarrow 2 h$ as well as four-body decays, e.g. $Y \rightarrow t \bar{t} t \bar{t}$ or $Y \rightarrow 4 h$, can do the job. The decay spectra will also be modified by EW corrections and this will be partially taken into account by us as described above (ii). In fact, these corrections, in general, tend to shift neutrino events to lower energies. As we will show in the work, even considering only (ii) $Y \rightarrow \nu_{\alpha} N$, we will also have a low energy tail due to EW corrections and red shift effect from extra-galactic contributions (see also [24]).

In sections 3-5, we will consider the LLP to be a scalar $Y$ with three possible decay channels: $Y \rightarrow \nu_{\alpha} N, Y \rightarrow 2 h$ and $Y \rightarrow 4 h$. Here we assume $N$ is a fermion singlet which is not a SM neutrino. If the reader is curious about its identity, he or she can go directly to section 6 where we present a consistent model which can realize such a particle with the required decay channels. For simplicity, we will assume that $Y$ decays with equal branching ratios to neutrinos of all flavours and suppress the flavour index in what follows, i.e. $Y \rightarrow \nu N$. For the decays $Y \rightarrow 2 h$ and $Y \rightarrow 4 h$, we use PYTHIA to generate the energy spectrum of the neutrinos taking into account of SM particles decay and hadronization. ${ }^{4}$ Then we consider neutrino oscillations to properly account for the neutrino flavours which arrive at the Earth. Finally before proceeding to data analysis, we want to stress that the above choice is by no means the unique decay channels for a LLP which can describe the suggestive features of the IceCube data but simply a working assumption.

\section{IceCube data analysis: a brief description}

We will consider in our calculations three different sources of neutrinos in IceCube: LLP decays, an unknown astrophysical source (modelled with a power-law spectrum) and cosmic ray air showers.

The contributions of LLP decays to the IceCube neutrino flux have two different components: a galactic and a diffuse extra-galactic contribution. The neutrino differential flux from galactic LLP decays can be calculated as [23]

$$
\frac{d \Phi_{\nu}}{d E_{\nu} d b d l}=\frac{1}{N} \frac{d N}{d E_{\nu}} \frac{1}{\tau_{Y} M_{Y}} \frac{\cos (b)}{4 \pi} \int d s \rho_{Y}(r(s))
$$

\footnotetext{
${ }^{4}$ The EW corrections to the neutrino energy spectrum are taken into account only for the decay channels $Y \rightarrow \nu N$ and $Y \rightarrow 2 h$ as described above (ii) while for the channel $Y \rightarrow 4 h$, since there is no straightforward way to implement such corrections, we ignore them keeping in mind that these corrections tend to shift neutrino events to lower energies.
} 
where the integral on $s$ is along the line of sight and $r^{2}=s^{2}+r_{0}^{2}-2 s r_{0} \cos (l) \cos (b)$, with $-90^{\circ} \leq b<90^{\circ}$ and $-180^{\circ} \leq l<180^{\circ}$. Here $r_{0}=8.5 \mathrm{kpc}$ is the distance from the Sun to the galactic center. The term $(1 / N) d N / d E_{\nu}$ is the normalized neutrino energy spectrum calculated using PYTHIA in the context of the LLP scenario proposed. For the galactic LLP matter density we use the DM Einasto density profile,

$$
\rho_{Y}(r)=\rho_{0} e^{-\frac{2}{\bar{\alpha}}\left[\left(\frac{r}{r_{s}}\right)^{\bar{\alpha}}-\left(\frac{r_{0}}{r_{s}}\right)^{\bar{\alpha}}\right]},
$$

with the standard choices $r_{s}=20 \mathrm{kpc}, \bar{\alpha}=0.17$ and $\rho_{0}=0.3 \mathrm{GeV} \mathrm{cm}^{-3}$. From this we can calculate the galactic neutrino flux contribution as

$$
\left(\frac{d \Phi_{\nu}}{d E_{\nu}}\right)_{\mathrm{gal}}=\left(\frac{1.3 \times 10^{-13}}{\mathrm{~cm}^{2} \mathrm{sr} \mathrm{s}}\right) \frac{10^{28} \mathrm{~s}}{\tau_{Y}} \frac{1 \mathrm{PeV}}{M_{Y}} \frac{1}{N} \frac{d N}{d E_{\nu}} .
$$

The extra-galactic contribution to the neutrino flux [44] is given by

$$
\frac{d \Phi_{\nu}}{d E_{\nu}}=\frac{\Omega_{\mathrm{DM}} \rho_{c}}{4 \pi \tau_{Y} M_{Y} H_{0} \Omega_{M}^{1 / 2}} \int_{1}^{\infty} d y \frac{d N}{N d\left(E_{\nu} y\right)} \frac{y^{-3 / 2}}{\sqrt{1+\left(\Omega_{\Lambda} / \Omega_{M}\right) y^{-3}}},
$$

where $y=1+z, z$ being the red-shift. This can also be written as

$$
\begin{aligned}
\left(\frac{d \Phi_{\nu}}{d E_{\nu}}\right)_{\mathrm{ex}-\mathrm{gal}}= & \left(\frac{2.5 \times 10^{-13}}{\mathrm{~cm}^{2} \mathrm{srs}}\right) \frac{10^{28} \mathrm{~s}}{\tau_{Y}} \frac{1 \mathrm{PeV}}{M_{Y}} \\
& \times \int_{1}^{\infty} d y \frac{d N}{N d\left(E_{\nu} y\right)} \frac{y^{-3 / 2}}{\sqrt{1+\left(\Omega_{\Lambda} / \Omega_{M}\right) y^{-3}}},
\end{aligned}
$$

where the numerical values of the cosmological densities $\Omega_{\mathrm{DM}}=0.265, \Omega_{M}=0.315$, $\Omega_{\Lambda}=0.685$, Hubble constant $H_{0}=67.3 \mathrm{~km} / \mathrm{s} / \mathrm{Mpc}$ and $\rho_{c}=1.054 \times 10^{-5} h^{2} \mathrm{GeV} / \mathrm{cm}^{3}$ were taken from $[45,59]$. Here we want to stress that although in the fit we have fixed $\Omega_{\mathrm{DM}}=0.265$ to be all the DM density, in principle the LLP can constitute only part of the DM. The lifetime of the LLP $\tau_{Y}$ obtained from the fit will have to be multiplied by a factor of $\kappa$ if the LLP constitute only a fraction $\kappa$ to the DM density. Hence $\tau_{Y}$ obtained from the fit is the upper bound on the lifetime of $Y$. We refer the reader to section 6.3 for a discussion on the LLP abundance. Again the term $(1 / N) d N / d E_{\nu}$ is the normalized neutrino energy spectrum calculated using PYTHIA in accordance to the LLP decay modes of a particular scenario. In the above $M_{Y}$ and $\tau_{Y}$ are parameters to be fit to the data.

The cosmic unknown neutrino source contribution was estimated as a power-law similar to $[36,46]$ but using the convenient parametrization

$$
\left(\frac{d \Phi_{\nu}}{d E_{\nu}}\right)_{\mathrm{pl}}=\frac{C_{0}}{10^{8}} \times \frac{1}{E_{\nu}^{2}} \times\left(\frac{E_{\nu}}{100 \mathrm{TeV}}\right)^{2-s}
$$

where $C_{0}$ is the per-flavour normalization $(1: 1: 1)$ and $s$ is the spectral index. These are parameters to be fit to the experimental observations. The total number of neutrino events expected in the $n$-th energy bin of IceCube, $N\left(E_{n}\right)$, is calculated as

$$
N\left(E_{n}\right)=T \times \Omega \times \sum_{j, \alpha} \int_{E_{n}}^{E_{n+1}} d E_{\nu} A_{\mathrm{eff}}^{\alpha}\left(E_{\nu}\right)\left(\frac{d \Phi_{\nu}}{d E_{\nu}}\right)_{j}^{\alpha},
$$


where $T$ is the exposure time, here 988 days [38], $\Omega=4 \pi$ is the solid angle of coverage, $A_{\text {eff }}^{\alpha}\left(E_{\nu}\right)$ is the effective area for the neutrino flavour $\alpha$ taken from [10], $E_{n}$ and $E_{n+1}$ are the lower and upper energy limits of the bin and the sum $\sum_{j, \alpha}$ is performed over each lepton flavour $\alpha$ and the different contributions to the neutrino flux (e.g. eqs. (3.3), (3.5), (3.6) and the atmospheric background neutrinos discussed in the next paragraph), labelled by $j$, for each scenario as shown in table 1 . For scenarios that contain two decaying channels the branching ratio $r_{\nu N}$ is included in the computation of $(1 / N) d N / d E_{\nu}$.

Finally we will also take into account the background neutrinos that arise from cosmic ray air showers, mainly from muon, $\pi / K$ and charm decays, simply by using the digitalized numbers for the atmospheric background from the IceCube paper [38]. We have extrapolated this background to higher energy bins (up to $10 \mathrm{PeV}$ ).

\section{Fitting the IceCube data in three scenarios}

We compare three scenarios in fitting the three years of IceCube data [38]: (a) a single power-law, (b) a power-law and a two-body LLP decay and (c) pure LLP decays according to the LLP model described in section 6 . In the analysis, we consider the energy domain from about $10 \mathrm{TeV}$ up to $10 \mathrm{PeV}$, altogether 14 bins as shown for e.g. in figure 1. Although the last three bins (from about $2 \mathrm{PeV}$ to $10 \mathrm{PeV}$ ) have null events, they are important to help to disentangle the various hypotheses as we will show in section 5 .

In the scenario (a) we have two free parameters, $s$ and $C_{0}$ (eq. (3.6)), in the scenario (b) we have an extra free parameter, the lifetime $\tau_{Y}$ while the LLP mass $M_{Y}$ is fixed to be either 2.2 or $4.0 \mathrm{PeV}$ and the branching ratio $r_{\nu N}=\mathrm{BR}(Y \rightarrow \nu N)=1$. Finally, in the scenario (c) we will also have two free parameters $\tau_{Y}$ and $r_{\nu N}$ while $M_{Y}$ will also be fixed to be either 2.2 or $4.0 \mathrm{PeV}$.

In order to estimate the best fit values of the parameters in each case we use the method of maximum likelihood by constructing a probability distribution function (pdf). We also compute the p-value associated with each hypothesis $\mathrm{H}_{0}$. The description of our statistical procedure can be found in appendix A.

The summary of best fit parameters and the corresponding p-values for the hypothesis considered in this work are given in table 1.

\subsection{Power-law fit $\left(\mathrm{H}_{0}=\mathrm{I}\right)$}

In figure 1 we show the best fit curve for an unbroken power-law spectrum fit to the data. Although it is at this point unclear if this power-law behaviour can be explained by a single astrophysical type of source, this hypothesis is the simplest one. We show the atmospheric background contribution (red curve), the power-law contribution (magenta curve) as well as the sum of the atmospheric background and the power-law signal fit (blue curve) for our best fit value at $s=2.3$ and $C_{0}=0.6 \mathrm{GeV} \mathrm{cm}^{-2} \mathrm{~s}^{-1} \mathrm{sr}^{-1}$, which corresponds to a p-value of 0.5 . From this we see that the power-law contribution is only significant for energies $\gtrsim 100 \mathrm{TeV}$, the lower energy part of the spectrum is dominated by the atmospheric background. If this hypothesis is true there should be events in the gap between $400 \mathrm{TeV}$ and $1 \mathrm{PeV}$ and above $2 \mathrm{PeV}$ in the future. 


\begin{tabular}{|c|c|c|c|c|c|c|c|c|}
\hline $\mathrm{H}_{0}$ & $M_{Y}[\mathrm{PeV}]$ & Scenario & $s$ & $C_{0}$ & $\tau_{Y} \times 10^{28}[\mathrm{~s}]$ & $r_{\nu N}$ & $\chi_{\min }^{2}$ & $p$ \\
\hline I & - & $\mathrm{PL}$ & 2.3 & 0.6 & - & - & 39.41 & 0.5 \\
\hline II.a & 2.2 & $\mathrm{PL}+\nu N$ & 2.43 & 0.51 & 5.26 & - & 38.07 & 0.45 \\
\hline II.b & 4.0 & $\mathrm{PL}+\nu N$ & 2.76 & 0.52 & 2.72 & - & 36.67 & 0.58 \\
\hline III.a & 2.2 & $\nu N+4 h$ & - & - & 0.73 & 0.14 & 42.53 & 0.06 \\
\hline III.b & 4.0 & $\nu N+4 h$ & - & - & 0.88 & 0.35 & 36.6 & 0.56 \\
\hline IV.a & 2.2 & $\nu N+2 h$ & - & - & 1.81 & 0.56 & 44.87 & 0.01 \\
\hline IV.b & 4.0 & $\nu N+2 h$ & - & - & 1.13 & 0.23 & 36.25 & 0.57 \\
\hline $\mathrm{V}$ & 4.0 & $\nu N$ & - & - & 1.9 & - & 38.64 & 0.24 \\
\hline
\end{tabular}

Table 1. Summary of best fit parameters and p-values for the hypothesis $\mathrm{H}_{0}$ considered in this work. PL stands for power-law, $C_{0}$ is given in $\mathrm{GeV} \mathrm{cm}^{-2} \mathrm{~s}^{-1} \mathrm{sr}^{-1}$ and $r_{\nu N}$ is the branching ratio of the channel $Y \rightarrow \nu N$.

-.- ATM … PL - TOT

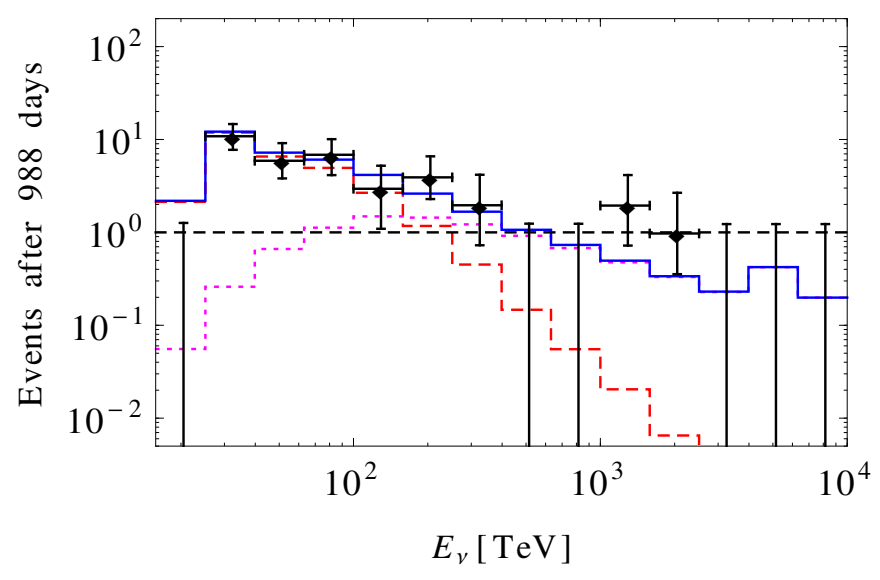

Figure 1. Best fit curve for an unbroken power-law spectrum with $s=2.3$ and $C_{0}=$ $0.6 \mathrm{GeV} \mathrm{cm}{ }^{-2} \mathrm{~s}^{-1} \mathrm{sr}^{-1}$. The IceCube data points (black crosses) are shown as well as the contributions from atmospheric background (ATM, red), the single power-law spectrum (PL, magenta) and the total contribution (TOT, blue).

An unbroken power-law spectrum such as this one may arise from optically thin galactic neutrino sources [16]. It has been pointed out that cosmic ray interactions with gas, such as expected around supernova remnants, seem to be able to produce smooth neutrino spectra [47].

Here a note is in order. Our best-fit value is compatible to IceCube spectral index fit [38] within $1 \sigma$ since at this confidence level $1.72 \leq s \leq 2.83$. We show in figure 2 the correlation between $C_{0}$ and the spectral index $s$ for our fit. We can see that a small change in $s$ can cause a significant change in $C_{0}$ and vice-versa, so the best fit values of these parameters are not at this point very significant.

Also we would like to comment on the new IceCube veto-based technique developed to study the neutrino spectrum between 10 and $100 \mathrm{TeV}$ [48]. Using this new method they 


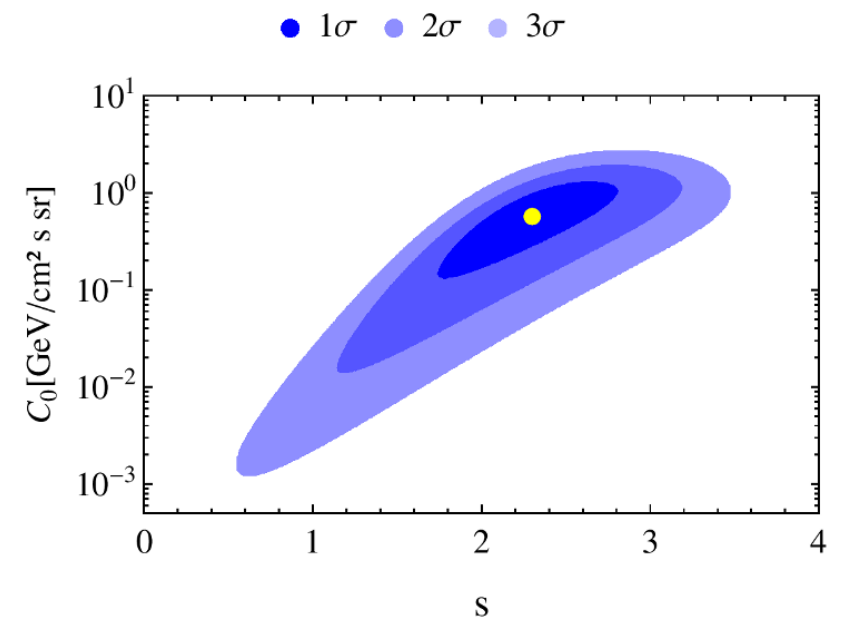

Figure 2. Contour plot of the allowed region in the plane $C_{0} \times s$ at 1,2 and $3 \sigma$ CL for the power-law hypothesis.

were able to better understand their background at lower energies. Although this could have an impact on the best fit values of the parameters in our analysis, we do not believe our conclusions would change. For completeness we present in table 4 in appendix B the 1,2 and $3 \sigma$ confidence intervals for $s$ and $C_{0}$ for our fit.

\subsection{Power-law + Long-Lived Particle two body decay fit $\left(\mathrm{H}_{0}=\mathrm{II}\right)$}

In figure 3 we show the best fit curve for a fit of the data with a contribution from a powerlaw spectrum combined with a contribution from the LLP decay $Y \rightarrow \nu N$. On the left panel we show the case $M_{Y}=2.2 \mathrm{PeV}$, for the best fit $s=2.43, C_{0}=0.51 \mathrm{GeV} \mathrm{cm}^{-2} \mathrm{sr}^{-1} \mathrm{~s}^{-1}$ and $\tau_{Y}=5.26 \times 10^{28} \mathrm{~s}$, corresponding to a p-value of 0.45 . On the right panel we show the case $M_{Y}=4 \mathrm{PeV}$, for the best fit $s=2.76, C_{0}=0.52 \mathrm{GeV} \mathrm{cm}^{-2} \mathrm{sr}^{-1} \mathrm{~s}^{-1}$ and $\tau_{Y}=2.72 \times 10^{28} \mathrm{~s}$, corresponding to a p-value of 0.58 . The hypotheses I, II.a and II.b all have very similar p-values and at this point seem to be indistinguishable. In both cases II.a and II.b, the power-law contribution is similar to the single power-law fit and the LLP decay basically contributes to the 1 or $2 \mathrm{PeV}$ energy bin, depending on $M_{Y}$. The future content of these bins can help to distinguish the hybrid hypothesis from the single power-law one.

The confidence intervals for the fitted parameters can also be found in table 4 in appendix B. From these intervals we see that if one allows for both a LLP two-body decay as well as a contribution from a power-law spectrum, there is a minimum lifetime for the LLP compatible with the data, but longer lifetimes are clearly also possible because in this case in practice we revert back to the single power-law case.

\subsection{Pure Long-Lived Particle decays fit $\left(\mathrm{H}_{0}=\right.$ III, IV and V)}

We have examined three scenarios for pure LLP decays. For $\mathrm{H}_{0}=$ III we have the two comparable decay modes: $Y \rightarrow \nu N$ and $Y \rightarrow 4 h$. For $\mathrm{H}_{0}=\mathrm{IV}$ we have two comparable two-body decay modes: $Y \rightarrow \nu N$ and $Y \rightarrow 2 h$, whereas for $\mathrm{H}_{0}=\mathrm{V}$ we have only a single decay contribution $Y \rightarrow \nu N$. 

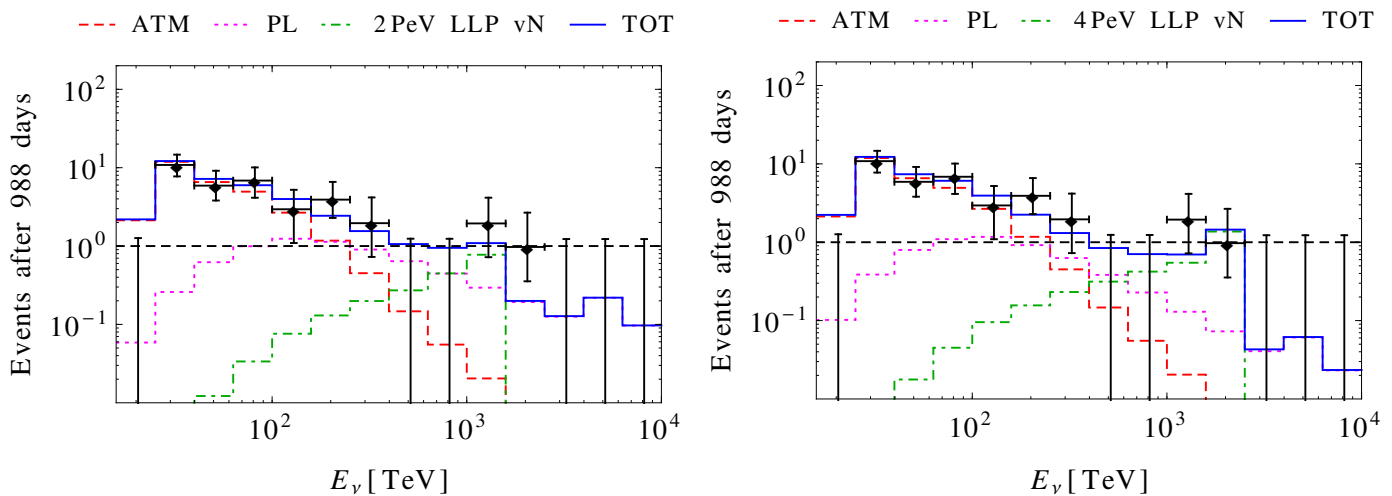

Figure 3. Best fit curve for an unbroken power-law spectrum combined with the LLP decay $Y \rightarrow \nu N$. The left panel is for $M_{Y}=2.2 \mathrm{PeV}, s=2.43, C_{0}=0.51 \mathrm{GeV} \mathrm{cm}^{-2} \mathrm{sr}^{-1} \mathrm{~s}^{-1}$ and $\tau_{Y}=5.26 \times 10^{28} \mathrm{~s}$. The right panel is for $M_{Y}=4 \mathrm{PeV}, s=2.76, C_{0}=0.52 \mathrm{GeV} \mathrm{cm}^{-2} \mathrm{sr}^{-1} \mathrm{~s}^{-1}$ and $\tau_{Y}=2.72 \times 10^{28} \mathrm{~s}$. The IceCube data points (black crosses) are shown as well as the contributions from atmospheric background (ATM, red), single power-law spectrum (PL, magenta), LLP decay (LLP, green) and the total contribution (TOT, blue).
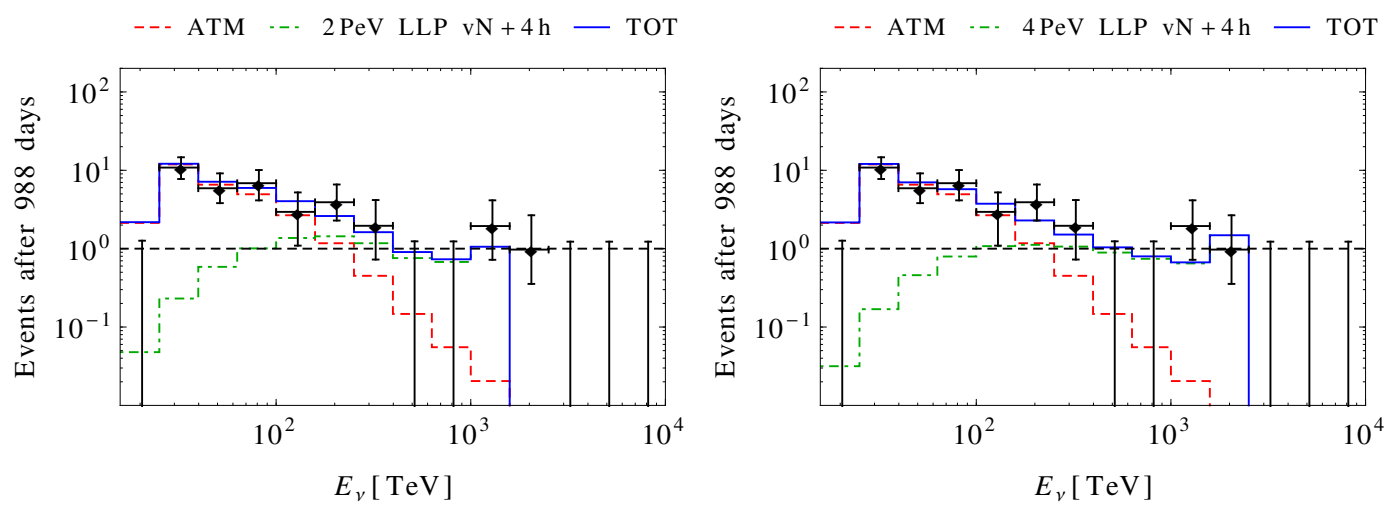

Figure 4. Best fit curve for pure LLP decays into $Y \rightarrow \nu N$ and $Y \rightarrow 4 h$. The left panel is for $M_{Y}=2.2 \mathrm{PeV}, \tau_{Y}=0.73 \times 10^{28} \mathrm{~s}$ and $r_{\nu N}=14 \%$. The right panel is for $M_{Y}=4 \mathrm{PeV}$, $\tau_{Y}=0.88 \times 10^{28} \mathrm{~s}$ and $r_{\nu N}=35 \%$. The IceCube data points (black crosses) are shown as well as the contributions from atmospheric background (ATM, red), LLP decays (LLP, green) and the total contribution (TOT, blue).

In figure 4 we show the best fit curve for pure LLP decay into the two modes $Y \rightarrow \nu N$ and $Y \rightarrow 4 h$. On the left panel we show the case $M_{Y}=2.2 \mathrm{PeV}$, for the best fit $\tau_{Y}=$ $0.73 \times 10^{28} \mathrm{~s}$ and $r_{\nu N}=0.14$, corresponding to a p-value of 0.06 . On the right panel we show the case $M_{Y}=4 \mathrm{PeV}$, for the best fit $\tau_{Y}=0.88 \times 10^{28} \mathrm{~s}$ and $r_{\nu N}=0.35$, corresponding to a p-value of 0.56 .

In figure 5 we show the best fit curve for pure LLP decay into the two modes $Y \rightarrow \nu N$ and $Y \rightarrow 2 h$. On the left panel we show the case $M_{Y}=2.2 \mathrm{PeV}$, for the best fit $\tau_{Y}=$ $1.81 \times 10^{28} \mathrm{~s}$ and $r_{\nu N}=0.56$, corresponding to a p-value of 0.01 . On the right panel we show the case $M_{Y}=4 \mathrm{PeV}$, for the best fit $\tau_{Y}=1.13 \times 10^{28} \mathrm{~s}$ and $r_{\nu N}=0.23$, corresponding to a p-value 0.57 . 

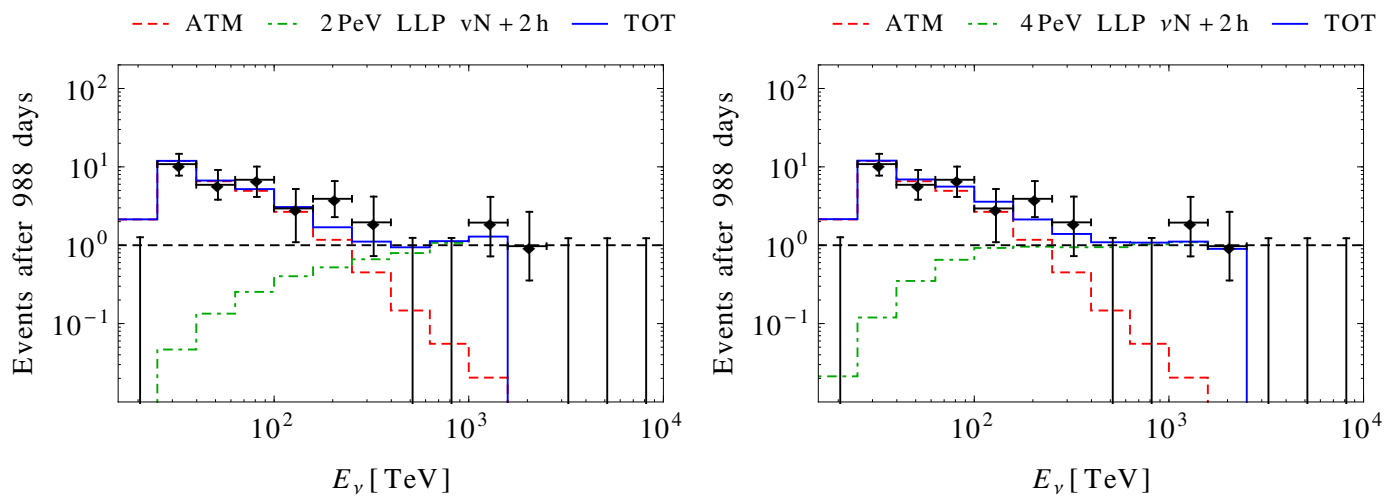

Figure 5. Best fit curve for pure LLP decays into $Y \rightarrow \nu N$ and $Y \rightarrow 2 h$. The left panel is for $M_{Y}=2.2 \mathrm{PeV}, \tau_{Y}=1.81 \times 10^{28} \mathrm{~s}$ and $r_{\nu N}=56 \%$. The right panel is for $M_{Y}=4 \mathrm{PeV}$, $\tau_{Y}=1.13 \times 10^{28} \mathrm{~s}$ and $r_{\nu N}=23 \%$. The IceCube data points (black crosses) are shown as well as the contributions from atmospheric background (ATM, red), LLP decays (LLP, green) and the total contribution (TOT, blue).
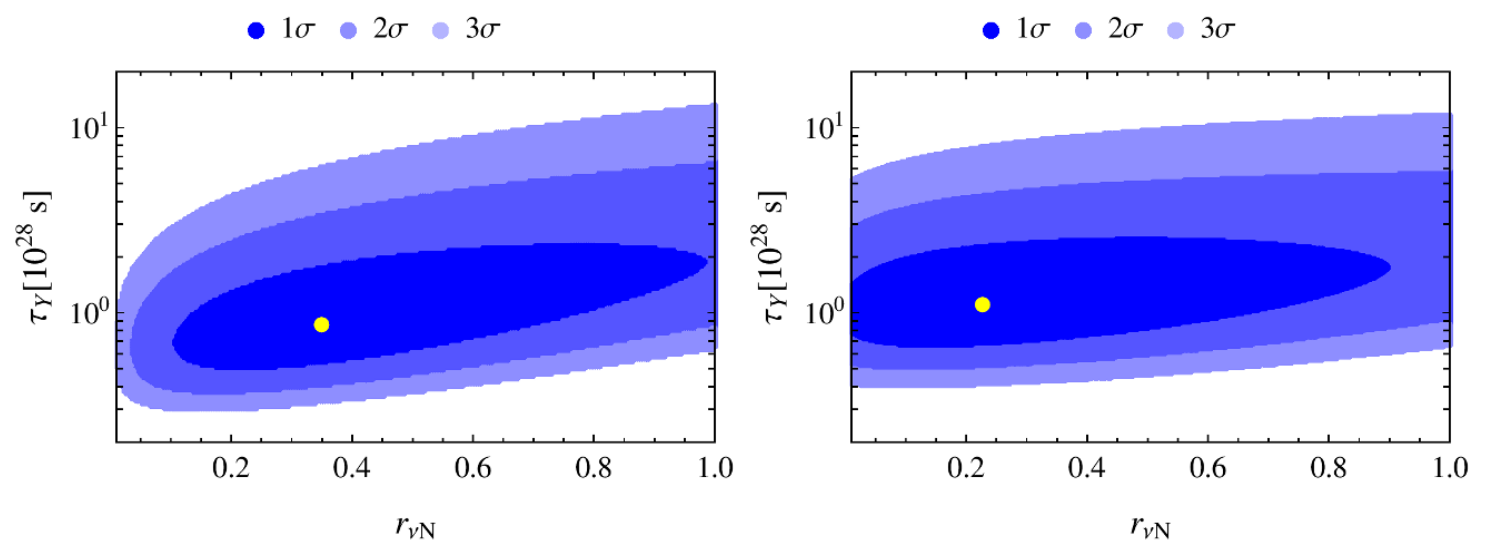

Figure 6. Contour plots of the allowed regions in the plane $\tau_{Y} \times r_{\nu N}$ at 1,2 and $3 \sigma$ CL for the hypotheses III.b (left panel) and IV.b (right panel).

Clearly the cases with $M_{Y}=2.2 \mathrm{PeV}$ are very unfavourable, but the cases with $M_{Y}=4 \mathrm{PeV}$ are consistent with data. In fact, the fit with $M_{Y}=4 \mathrm{PeV}$ and the LLP decaying into $\nu N$ and $2 h$ presents one of the highest p-value of all cases studied. We see that the LLP decays start to contribute to the spectrum at energies $\gtrsim 70 \mathrm{TeV}$ up to $2 \mathrm{PeV}$, so these scenarios predicts a sharp cutoff in the spectrum above $2 \mathrm{PeV}$ that could be confirmed by future data.

In figure 6 we show the correlation between $\tau_{Y}$ and the branching ratio $r_{\nu N}$ for $M_{Y}=4 \mathrm{PeV}$ and hypotheses III and IV. We see the $Y$ lifetime is slightly correlated with $r_{\nu N}$, more so for III than for IV. Also when we have $Y \rightarrow 2 h$ it is possible, even at $1 \sigma$, to have $r_{\nu N}=0$ while when we have $Y \rightarrow 4 h$ we need at least some $Y \rightarrow \nu N$ contribution in order to explain the data.

Finally, we also investigate the single decay mode $Y \rightarrow \nu N$ for $M_{Y}=4 \mathrm{PeV}$ for which we get the best fit $\tau_{Y}=1.9 \times 10^{28} \mathrm{~s}$ and a corresponding p-value of 0.25 . This case is shown 


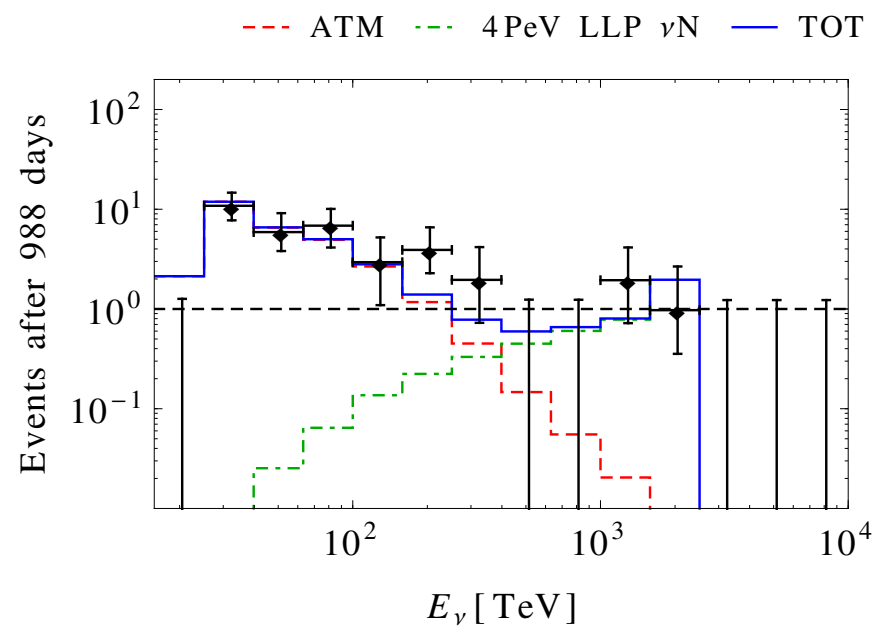

Figure 7. Best fit curve for a $M_{Y}=4 \mathrm{PeV}$ LLP decaying into $Y \rightarrow \nu N$ with $\tau_{Y}=1.9 \times 10^{28} \mathrm{~s}$. The IceCube data points (black crosses) are shown as well as the contributions from atmospheric background (ATM, red), the LLP decay (LLP, green) and the total contribution (TOT, blue).

in figure 7 . We see that in this case instead of just a peak at $2 \mathrm{PeV}$, there is a cascade tail of lower energies neutrinos due to EW corrections (partially also due to the extragalactic neutrinos from LLP decay that red-shift to lower energies). Nevertheless the LLP decay only starts to contribute at much higher energies, around $500 \mathrm{TeV}$, so up to that point the spectrum has to be entirely explained by the atmospheric background. Also this scenario predicts a cutoff in the spectrum after $2 \mathrm{PeV}$. Just by looking at figure 7 we see that the data in the two bins bellow $500 \mathrm{TeV}$ and the bin at $1 \mathrm{PeV}$ seem to be higher than the theoretical prediction. You can find in table 4 in appendix B the allowed intervals of the parameters for all cases considered here.

To conclude this section we note that at this point the data seems to be equally compatible with a single power-law spectrum, a power-law plus $Y \rightarrow \nu N$ spectrum or a spectrum due to a $4 \mathrm{PeV}$ LLP decaying into $\nu N$ and $2 h$ or $4 h$.

\subsection{Constraints from gamma-ray and antiproton observations}

We now briefly discuss the question of whether our LLP decay scenario for IceCube highenergy events is consistent with the limits imposed by diffuse gamma-ray and antiproton observations. Here, we limit ourselves to a sketchy description by just reviewing the results in the existing literatures. ${ }^{5}$ As discussed by the authors of [49], the cascade gamma-ray bound is largely DM mass-independent at sufficiently high masses, because it is essentially bolometric in nature. It allows us relatively DM mass independent conclusion. Also the gamma-ray limits at very high masses is weaker than the limit for neutrinos which was obtained [49] assuming non observation of three years run of IceCube. When applied to our case, it means that models of LLP decay which explains IceCube neutrino excess would

\footnotetext{
${ }^{5}$ We note that the limit derived for super-heavy DM applies to our LLP scenario because the mass density of LLP cannot exceeds that of DM. That is, the DM mass density gives a maximum possible value of LLP mass density, and hence if DM evades a limit our LLP does.
} 


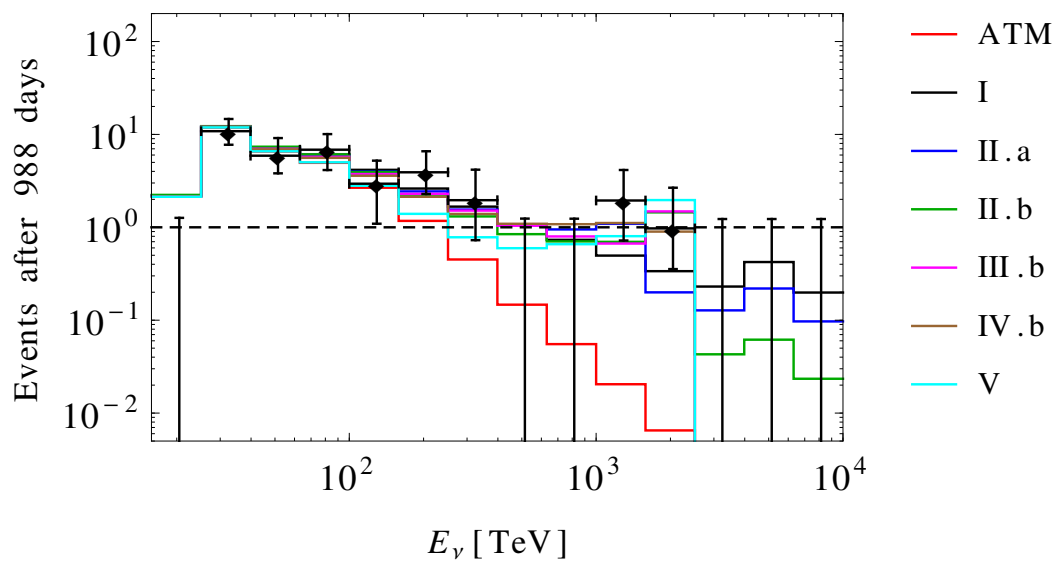

Figure 8. The best fit for all the hypotheses with the current data and the atmospheric background extrapolated up to the energy range $[10 \mathrm{TeV}, 10 \mathrm{PeV}]$.

be free from the diffuse gamma ray bound, even though the new Fermi-LAT data at higher energies [50] makes the consistency more nontrivial [51]. The authors of [27] also reached the similar conclusion on gamma ray bound with the recent Fermi data.

More specifically in our case, the model we examined in this section is much safer than the generic LLP decay scenario, because the decay products, neutrinos, gammas, and electrons, etc. from Higgs boson is about 10 times less prominent compared to those from $b \bar{b}$ at low energies (see also [19]). It appears that the PAMELA antiproton limit is also cleared by our LLP scenario, as one can see in figure 6 in [52]. By extrapolating three-orders of magnitude in the DM mass from those in figure 6 the lifetime lower bound is well below $10^{27} \mathrm{~s}$.

\section{$5 \quad$ Future data perspectives}

In view of the fact that at this point the data seems to be compatible with many different cases we would like to establish the necessary exposure time in order to exclude a given hypothesis $\mathrm{H}_{\mathrm{j}}$ assuming the true explanation of the data is $\mathrm{H}_{\mathrm{i}}$. We refer the reader to appendix A for details of the statistical calculation. Our discussion in this section relies only on energy spectrum information. ${ }^{6}$

In figure 8 we show the curves for all the hypotheses best fit with the current data. From this we can clearly see that data at higher energies will be able to help to disentangle the various hypotheses.

In table 2 we show the results of our computations for all possible combinations of the hypotheses we consider. In the columns we place the true hypothesis $H_{i}$ while in the rows we place the hypothesis to be excluded $H_{j}$. We define the exclusion time as $T=f_{T} \times 988$ days. In each cell we write the vector $\left(f_{T}, \mathrm{p} \times 100\right)$ in order to indicate the necessary exposure time and the corresponding $\mathrm{p}$-value as a percentage computed at that time. Also, we use the

\footnotetext{
${ }^{6}$ There may be other ways to distinguish the various hypotheses including ours, for example, by pinning down the sources, possible identification of galactic-extragalactic components, and by correlating with gamma ray observation.
} 


\begin{tabular}{|c|c|c|c|c|c|c|}
\cline { 2 - 7 } \multicolumn{1}{c|}{} & I & II.a & II.b & III.b & IV.b & V \\
\hline I & - & $(25,3)^{*}$ & $(8,3)$ & $(8,2)$ & $(9,2)$ & $(4.5,3)$ \\
\hline II.a & $(100,100)^{* *}$ & - & $(7,3)$ & $(8,2)$ & $(12,5)$ & $(4.5,2)$ \\
\hline II.b & $(100,100)^{* *}$ & $(23,3)^{*}$ & - & $(75,4)^{*}$ & $(25,4)^{*}$ & $(100,100)^{* *}$ \\
\hline III.b & $(4.5,2)$ & $(6,3)$ & $(34,1)^{*}$ & - & $(28,5)^{*}$ & $(100,100)^{* *}$ \\
\hline IV.b & $(4.5,1)$ & $(9,4)$ & $(20,4)^{*}$ & $(45,5)^{*}$ & - & $(100,100)^{* *}$ \\
\hline V & $(2.5,1)$ & $(3.5,4)$ & $(12,4)$ & $(11,4)$ & $(9,3)$ & - \\
\hline
\end{tabular}

Table 2. Estimation of the exclusion time needed to eliminate a hypothesis. In each cell we write the vector $\left(f_{T}, \mathrm{p} \times 100\right)$. We use the notation $\left(f_{T}, \mathrm{p} \times 100\right)^{*}$ to identify combinations that can be excluded but in a very long time and $\left(f_{T}, \mathrm{p} \times 100\right)^{* *}$ for those combinations that cannot be excluded.

notation $\left(f_{T}, \mathrm{p} \times 100\right)^{*}$ to identify combinations that can be distinguish but in a very long time and $\left(f_{T}, \mathrm{p} \times 100\right)^{* *}$ for those combinations that cannot be distinguished at any time.

We see that assuming a power-law spectrum, the solely LLP decay models can be excluded with two to five times the current data due to the absence of neutrino events beyond the cutoff energy. If we introduce a LLP component to the power-law it will take a longer time to distinguish from the solely LLP decay models because in this case the power-law spectral index turns out to be larger and predicts less events beyond the cutoff. A single power-law and a power-law plus the LLP decay is rather difficult to distinguish unless one constraints the lifetime of the LLP to a maximum value. If the future data is consistent with solely LLP decays it will be difficult to distinguish among the possible contributing decay mode scenarios, but the sharp cutoff in the spectrum will certainly indicate a LLP decay component.

\section{A model for a Long-Lived Particle}

In this section we present a consistent model for a LLP that could give rise to the decay channels used to fit the data in the previous sections. We must emphasize that although we rely on this particular model to fit the IceCube high-energy excess events, it is by no means the unique model that can explain the IceCube data with or without the power-law component. However, we hope that it serves as the existence proof of such models that can explain the data only by LLP decays.

As seen in the previous sections, we can accommodate the IceCube data with the following decay channels: $Y \rightarrow \nu N, Y \rightarrow 2 h$ and $Y \rightarrow 4 h$. To accomplish this, we introduce two complex scalar fields, $Y$ and $X$, that are singlets under the SM gauge group. For the fermionic sector, we introduce a new vectorlike pair of fermion doublets $\Psi_{L}=\left(\psi_{L}^{0}, \psi_{L}^{-}\right)^{T}$ and $\Psi_{R}=\left(\psi_{R}^{0}, \psi_{R}^{-}\right)^{T}$, and another right-handed fermion singlet $N_{R}$. This model has no SM gauge anomaly since $\Psi_{L}$ and $\Psi_{R}$ are vectorlike under the SM gauge interactions while $N_{R}$ is a singlet. In addition, we assume these new fields to be charged under a new $\mathrm{U}(1)_{X}$ symmetry according to table 3 . In the following we will consider both possibilities that 


\begin{tabular}{|c|c|c|c|}
\hline New fields & $\mathrm{SU}(2)_{L}$ & $\mathrm{U}(1)_{Y}$ & $\mathrm{U}(1)_{X}$ \\
\hline$X$ & 1 & 0 & 1 \\
\hline$Y$ & 1 & 0 & -2 \\
\hline$\Psi_{L}$ & 2 & $-1 / 2$ & 2 \\
\hline$\Psi_{R}$ & 2 & $-1 / 2$ & 2 \\
\hline$N_{R}$ & 1 & 0 & 2 \\
\hline
\end{tabular}

Table 3. New fields of the model and their respective assignments under $\mathrm{SU}(2)_{L} \times \mathrm{U}(1)_{Y} \times \mathrm{U}(1)_{X}$.

$\mathrm{U}(1)_{X}$ is global or local, keeping in mind that if $\mathrm{U}(1)_{X}$ is gauged, we need to introduce $N_{L}$ to cancel the $\mathrm{U}(1)_{X}$ gauge anomaly.

\subsection{The scalar sector}

Now we will describe the scalar sector of our model with the following scalar potential

$$
\begin{aligned}
V(X, Y, H)= & \frac{1}{4} \lambda_{X}\left(X^{\dagger} X-w^{2}\right)^{2}+\frac{1}{4} \lambda_{H}\left(H^{\dagger} H-v^{2}\right)^{2} \\
& +\frac{1}{4} \lambda_{Y}\left(Y^{\dagger} Y\right)^{2}+M_{Y}^{2} Y^{\dagger} Y \\
& +\lambda_{H X}\left(H^{\dagger} H-v^{2}\right)\left(X^{\dagger} X-w^{2}\right)+\lambda_{X Y}\left(X^{\dagger} X-w^{2}\right) Y^{\dagger} Y \\
& +\lambda_{H Y}\left(H^{\dagger} H-v^{2}\right) Y^{\dagger} Y-\left(\mu_{X Y} X X Y+\text { H.c. }\right),
\end{aligned}
$$

where $H$ is the SM Higgs doublet. We assume that all the dimensionless couplings $\lambda$ 's are positive. The complex dimension one coupling $\mu_{X Y}$ can be made real by redefining $X$ and/or $Y$ fields. Without loss of generality, in the following, $\mu_{X Y}$ is taken to be real and positive. We further assume that the new physics scale $\langle X\rangle=w \gg v=174 \mathrm{GeV}$. As we will see below, the LLP is approximately $Y_{R}$ (the real part of $Y$ ) and hence $M_{Y}$ is at the $\mathrm{PeV}$ scale. We also take $M_{Y}^{2}>0$ such that no large vacuum expectation value (vev) will be induced for $Y$. Instead, a small vev for $Y$ is induced through the $\mu_{X Y}$ term:

$$
\langle Y\rangle=u=\mu_{X Y} w^{2} / M_{Y}^{2},
$$

where we have assumed the condition $\mu_{X Y} w^{2} / M_{Y}^{3} \ll 1$. As we will see later, this condition is always fulfilled due to the longevity requirement of the LLP. In fact if $\mu_{X Y} \rightarrow 0$, there will be a $Z_{2}$ symmetry such that: $\Psi_{L, R} \rightarrow-\Psi_{L, R}$ and $Y \rightarrow-Y$ (see eq. (6.15)). Hence $\mu_{X Y}$ controls the lifetime of our LLP. A small $\mu_{X Y}$ is technically natural since in the limit $\mu_{X Y} \rightarrow 0$, there is an enhanced symmetry $\mathrm{U}(1)^{2}$ which corresponds to independent phase rotations of $X$ and $Y$.

$\mathrm{U}(1)_{X}$ is spontaneously broken when $X$ acquires a vev $w$. If the $\mathrm{U}(1)_{X}$ is a global symmetry, we will have one massless Nambu-Goldstone boson (NGB). According to ref. [53], if one considers $10^{9} \mathrm{GeV} \lesssim w \lesssim 10^{12} \mathrm{GeV}$, the NGB will decouple much before the neutrino decoupling temperature $T \sim \mathrm{MeV}$ and hence its temperature will red-shift to a much lower 
value and have small contribution to the energy density at the time of nucleosynthesis. Assuming the SM degrees of freedom, it is possible to have up to 37 NGBs. Alternatively, $\mathrm{U}(1)_{X}$ can be gauged and instead of a NGB, we will have a massive gauge boson associated with $\mathrm{U}(1)_{X}$. In this case the $w$ scale can be relaxed to a lower value.

In the following we write $Y=u+\left(Y_{R}+i Y_{I}\right) / \sqrt{2}, X=w+\left(X_{R}+i X_{I}\right) / \sqrt{2}, H=$ $v+h / \sqrt{2}$. Then the mass matrices for the fields $\left\{h, Y_{R}, X_{R}\right\}$ and $\left\{Y_{I}, X_{I}\right\}$ are respectively

$$
M_{R}^{2}=\left(\begin{array}{ccc}
\lambda_{H} v^{2}+\lambda_{H Y} u^{2} & 2 \lambda_{H Y} u v & 2 \lambda_{H X} v w \\
2 \lambda_{H Y} u v & M_{Y}^{2}+\frac{3}{2} \lambda_{Y} u^{2} & 2\left(\lambda_{X Y} u-\mu_{X Y}\right) w \\
2 \lambda_{H X} v w & 2\left(\lambda_{X Y} u-\mu_{X Y}\right) w & \lambda_{X} w^{2}+\left(\lambda_{X Y} u-2 \mu_{X Y}\right) u
\end{array}\right),
$$

and

$$
M_{I}^{2}=\left(\begin{array}{cc}
M_{Y}^{2}+\frac{1}{2} \lambda_{Y} u^{2} & 2 \mu_{X Y} w \\
2 \mu_{X Y} w & \left(\lambda_{X Y} u+2 \mu_{X Y}\right) u
\end{array}\right) .
$$

The longevity of LLP requires tiny $\mu_{X Y}$, which from eq. (6.2) implies small mixing between $Y_{R}$ and $X_{R}, h$ and $Y_{R}$, and $Y_{I}$ and $X_{I}$. On the other hand, the mixing between $h$ and $X_{R}$ is controlled by the ratio

$$
\delta_{H X} \equiv \frac{4 \lambda_{H X}^{2}}{\lambda_{H} \lambda_{X}}
$$

For the SM Higgs boson mass $M_{h}=125 \mathrm{GeV}$ the still allowed branching ratio for the Higgs invisible decay width is in the ball-park of $20 \%$ [54]. In our model, we have

$$
\Gamma\left(h \rightarrow X_{I} X_{I}\right)=\frac{\lambda_{H X}^{2} v^{2}}{32 \pi M_{h}},
$$

and constraining $\mathrm{BR}\left(h \rightarrow X_{I} X_{I}\right) \lesssim 0.2$, we obtain

$$
\lambda_{H X} \lesssim 0.01
$$

On the other hand, for a gauged $\mathrm{U}(1)_{X}$, there will be no such decay channel since the gauge boson associated with $\mathrm{U}(1)_{X}$ breaking is much heavier than the SM Higgs with its mass given by

$$
M_{h}^{2}=\lambda_{H}\left(1+\delta_{H X}\right) v^{2} .
$$

For simplicity, we assume $\delta_{H X} \ll 1$ such that the scalars $Y_{R}, Y_{I}, X_{R}$ and $X_{I}$ are approximately mass eigenstates with respective masses

$$
M_{Y_{R}}^{2}=M_{Y_{I}}^{2}=M_{Y}^{2}, \quad M_{X_{R}}^{2}=\lambda_{X} w^{2}, \quad M_{X_{I}}^{2}=0 .
$$

We assume $M_{X_{R}} \gg M_{Y_{R}}$ such that $Y_{R}$ cannot decay to $X_{R}$ but it can decay directly to $2 h$ or indirectly to $4 h$ through two off-shell $X_{R}$. In the following, we will drop the subscript $R$ for the LLP $Y_{R}$ and simply denote it as $Y$. From the context, there should be no confusion with the original complex field $Y$. 
The decay widths for the decays of $Y$ to scalars are given by

$$
\begin{aligned}
\Gamma\left(Y \rightarrow X_{I} X_{I}\right) & =\frac{1}{32 \pi} \frac{\left(\lambda_{X Y} u+\mu_{X Y}\right)^{2}}{M_{Y}}, \\
\Gamma(Y \rightarrow 2 h) & =\frac{\lambda_{H Y}^{2}}{32 \pi} \frac{u^{2}}{M_{Y}}, \\
\Gamma(Y \rightarrow 4 h) & \approx \frac{\lambda_{H X}^{4}}{16384 \pi^{5}}\left(\frac{\lambda_{X Y} u-\mu_{X Y}}{\lambda_{X}}\right)^{2} \frac{M_{Y}^{3}}{M_{X_{R}}^{4}} .
\end{aligned}
$$

Comparing the decay rates, we have

$$
\begin{aligned}
\frac{\Gamma(Y \rightarrow 4 h)}{\Gamma\left(Y \rightarrow X_{I} X_{I}\right)} & =\frac{1}{512 \pi^{4}}\left(\frac{\lambda_{H X}}{\lambda_{X}}\right)^{4}\left(\frac{\lambda_{X Y}-r_{Y}^{2}}{\lambda_{X Y}+r_{Y}^{2}}\right)^{2} r_{Y}^{4}, \\
\frac{\Gamma(Y \rightarrow 4 h)}{\Gamma(Y \rightarrow 2 h)} & =\frac{1}{512 \pi^{4}}\left(\frac{\lambda_{H X}}{\lambda_{X}}\right)^{4}\left(\frac{\lambda_{X Y}-r_{Y}^{2}}{\lambda_{H Y}}\right)^{2} r_{Y}^{4},
\end{aligned}
$$

where we define $r_{Y} \equiv M_{Y} / w$ and we have used eq. (6.9) for the mass $M_{X_{R}}$ and also eq. (6.2). Taking $10^{9} \mathrm{GeV} \lesssim w \lesssim 10^{12} \mathrm{GeV}$ and $M_{Y} \sim 10^{6} \mathrm{GeV}$, we have $10^{-6} \lesssim r_{Y} \lesssim 10^{-3}$. With the assumption $M_{X_{R}} \gg M_{Y}$, we have $\lambda_{X} \gg r_{Y}^{2}$. However with the assumption of small $H-X$ mixing $\delta_{H X} \ll 1$, we have $\lambda_{H X} / \lambda_{X} \ll \lambda_{H} /\left(4 \lambda_{H X}\right) \simeq M_{h}^{2} /\left(4 \lambda_{H X} v^{2}\right)$. Taking the maximum allowed value $\lambda_{H X}=10^{-2}$ from eq. (6.7), we have $\lambda_{H X} / \lambda_{X} \ll 13$. As we will see later in section 6.3, under reasonable assumptions, $\lambda_{H Y}$ and $\lambda_{X Y}$ are required to be small, $\lesssim 10^{-10}$, in order not to over-produce $Y$. Taking all the above considerations into account, we can write down conservative upper bounds

$$
\frac{\Gamma(Y \rightarrow 4 h)}{\Gamma\left(Y \rightarrow X_{I} X_{I}\right)} \lesssim 6 \times 10^{-13}, \quad \frac{\Gamma(Y \rightarrow 4 h)}{\Gamma(Y \rightarrow 2 h)} \lesssim 6 \times 10^{-13}\left(\frac{\lambda_{X Y}-r_{Y}^{2}}{\lambda_{H Y}}\right)^{2} .
$$

From the above, we see that in order to have $\Gamma(Y \rightarrow 4 h)>\Gamma(Y \rightarrow 2 h)$, we need a coupling $\lambda_{H Y} \lesssim 7 \times 10^{-7}\left|\lambda_{X Y}-r_{Y}^{2}\right|$. In addition, we also notice that the decay channel of $Y$ to the NGB $X_{I}$ always dominates over the channel $Y \rightarrow 4 h$ while it is generally faster than $Y \rightarrow 2 h$ unless $\lambda_{H Y}>\left|\lambda_{X Y}+r_{Y}^{2}\right|$. Hence in the global $\mathrm{U}(1)_{X}$ scenario, this channel $Y \rightarrow X_{I} X_{I}$ is usually the one which determines the lifetime of $Y{ }^{7}$ Requiring $\tau_{Y}>t_{0} \simeq 4.4 \times 10^{17} \mathrm{~s}$, we obtain using eq. (6.10)

$$
\mu_{X Y} \lesssim 1.2 \times 10^{-17}\left(1+\frac{\lambda_{X Y}}{r_{Y}^{2}}\right)^{-1}\left(\frac{M_{Y}}{10^{6} \mathrm{GeV}}\right)^{1 / 2} \mathrm{GeV}
$$

The constraint above does not apply in the gauged $\mathrm{U}(1)_{X}$ scenario since the $\mathrm{U}(1)_{X}$ gauge boson is assumed to be a lot heavier than $Y$. From section 4, we see that the lifetimes which fit the data fall in the range $\tau_{Y} \sim 10^{27-29} \mathrm{~s}$, assuring $Y$ to be long-lived. In this case, we can constrain $\mu_{X Y}$ using eq. (6.11) and obtain

$$
\mu_{X Y} \lesssim 2.6 \times 10^{-19}\left(\frac{M_{Y}}{10^{6} \mathrm{GeV}}\right)^{5 / 2}\left(\frac{10^{10} \mathrm{GeV}}{w}\right)^{2}\left(\frac{10^{-11}}{\lambda_{H Y}}\right) \mathrm{GeV}
$$

\footnotetext{
${ }^{7}$ In this case, since $Y$ has additional decay channel to invisible NGBs, the LLP lifetime $\tau_{Y}$ in the fit of the neutrino flux in the previous sections will be the partial lifetime of $Y$.
} 
The fact that the bound (6.14) is smaller than the bound (6.13) and not the other way around is interesting and also crucial. It implies that in the global $\mathrm{U}(1)_{X}$ scenario, if we can fit the neutrino flux to explain the IceCube excess, despite having a dominant decay channel of $Y$ to NGB, the longevity requirement on $Y$ is automatically fulfilled. In all cases, we see that $\mu_{X Y}$ is constrained to be very small and the condition in obtaining the induced vev (6.2) is always valid.

\subsection{The fermionic sector}

Now we describe the new fermionic sector of our model. With the introduction of a pair of vectorlike fermion doublets $\Psi_{L}, \Psi_{R}$ and a right-handed singlet $N_{R}$, we have the following new terms

$$
-\mathcal{L} \supset\left(y_{\Psi} \overline{\ell_{L}} \Psi_{R} Y+y_{\nu} \overline{\Psi_{L}} \widetilde{H} N_{R}+M_{\Psi} \overline{\Psi_{L}} \Psi_{R}+\text { H.c. }\right),
$$

where $\ell_{L}=\left(\nu_{L}, e_{L}\right)^{T}$ are the SM lepton doublets with the flavour index suppressed and $\widetilde{H}=i \sigma_{2} H^{*}$ with $\sigma_{2}$ the Pauli matrix. We assume that $M_{\Psi}>\mathrm{PeV}$ such that $Y$ cannot decay into it. After EW symmetry breaking, a mixing of the new fermions with the SM leptons will be induced such that

$$
\mathcal{L}_{m}=\left(\overline{e_{L}} \overline{\psi_{L}^{-}}\right) m_{e \Psi}\left(\begin{array}{c}
e_{R} \\
\psi_{R}^{-}
\end{array}\right)+\left(\overline{\nu_{L}} \overline{\psi_{L}^{0}}\right) m_{\nu \Psi}\left(\begin{array}{c}
N_{R} \\
\psi_{R}^{0}
\end{array}\right)+\text { H.c. },
$$

where we have defined the mass matrices

$$
\begin{aligned}
& m_{e \Psi}=\left(\begin{array}{cc}
y_{e} v & y_{\Psi} u \\
0_{1 \times 3} & M_{\Psi}
\end{array}\right), \\
& m_{\nu \Psi}=\left(\begin{array}{cc}
0_{3 \times 1} & y_{\Psi} u \\
y_{\nu} v & M_{\Psi}
\end{array}\right),
\end{aligned}
$$

with $y_{e}$ the $3 \times 3$ SM charged lepton Yukawa matrix and $y_{\Psi}$ a 3 -column vector. Without loss of generality, we can choose a basis where $y_{e}=\hat{y}_{e}$ is diagonal and real. Diagonalizing the mass matrix for charged leptons above, due to the very small $u / M_{\Psi}$, the charged leptons mass eigenstates are still $m_{\alpha} \equiv\left(\hat{y}_{e}\right)_{\alpha \alpha} v$ to a good approximation. For the neutral leptons, since we have introduced only one $N_{R}$, there is only one massive active neutrino with Dirac mass given by

$$
m_{\nu}=\sqrt{\sum_{\alpha}\left|\left(y_{\Psi}\right)_{\alpha}\right|^{2}} u \frac{y_{\nu} v}{M_{\Psi}} .
$$

As we will see shortly, the longevity of $Y$ implies an extremely small contribution to neutrino mass. This could be easily modified to accommodate the neutrino oscillation data for example by introducing two other heavy right-handed SM singlets uncharged under $\mathrm{U}(1)_{X}$ or more generally by introducing a dimension five Weinberg operator [55]. Since this is not directly relevant for our current study, we won't pursue it further.

Next the leptonic decay widths of $Y$ to charged and neutral leptons are, respectively,

$$
\Gamma\left(Y \rightarrow e_{\alpha} \overline{e_{\beta}}\right)=\frac{1}{32 \pi}\left|\left(y_{\Psi}\right)_{\alpha}\right|^{2}\left|\left(y_{\Psi}\right)_{\beta}\right|^{2} \frac{u^{2} m_{\beta}^{2}}{M_{\Psi}^{4}} M_{Y},
$$


and

$$
\Gamma\left(Y \rightarrow \nu_{\alpha} \overline{N_{R}}\right)=\frac{1}{32 \pi}\left|\left(y_{\Psi}\right)_{\alpha}\right|^{2} \frac{\left|y_{\nu}\right|^{2} v^{2}}{M_{\Psi}^{2}} M_{Y} .
$$

Taking the ratio of these widths we have

$$
\frac{\Gamma\left(Y \rightarrow e_{\alpha} \overline{e_{\beta}}\right)}{\Gamma\left(Y \rightarrow \nu_{\delta} \overline{N_{R}}\right)}=\left|\frac{\left(y_{\Psi}\right)_{\alpha}}{\left(y_{\Psi}\right)_{\delta}}\right|^{2}\left|\frac{\left(y_{\Psi}\right)_{\beta}}{y_{\nu}}\right|^{2}\left(\frac{u}{M_{\Psi}}\right)^{2}\left(\frac{m_{\alpha}}{v}\right)^{2} .
$$

Assuming the dimensionless couplings are of the same order and considering, for example, $m_{\tau} / v$, the ratio above is still suppressed by very small $u / M_{\Psi}$. Hence the decays of $Y$ into neutrinos will always largely dominate over the decays into charged leptons and the latter can be ignored.

Using eq. (6.19), the total decay width of $Y$ to neutrinos can be rewritten as

$$
\sum_{\alpha} \Gamma\left(Y \rightarrow \nu_{\alpha} \overline{N_{R}}\right)=\frac{1}{32 \pi} \frac{m_{\nu}^{2}}{u^{2}} M_{Y} .
$$

Requiring the lifetime of $Y$ to be longer than the age of the Universe $t_{0}$, we have

$$
\frac{m_{\nu}}{u}=\frac{m_{\nu} r_{Y}^{2}}{\mu_{X Y}} \lesssim 10^{-23}
$$

In order to maximize the contribution to neutrino mass, we take for instance $r_{Y}=10^{-6}$ and $\mu_{X Y}=10^{-17} \mathrm{GeV}$ (see eqs. (6.13) and (6.14)), we still only have $m_{\nu} \lesssim 10^{-19} \mathrm{eV}$. We can also translate the bound (6.22) into a bound for the dimensionless couplings using eq. (6.19) as follows

$$
\sqrt{\sum_{\alpha}\left|\left(y_{\Psi}\right)_{\alpha}\right|^{2}} y_{\nu} \lesssim 6 \times 10^{-20}\left(\frac{M_{\Psi}}{10^{6} \mathrm{GeV}}\right) .
$$

As we will see in section 6.3 , for $M_{\Psi} \sim \mathrm{PeV}$, we need $\left(y_{\Psi}\right)_{\alpha} \lesssim 10^{-11}$ in order not to over-produce $Y$ and this in turn implies $y_{\nu} \lesssim 10^{-9}$.

Finally, we would like to comment on possible phenomenological constraints on the fermion sector. In ref. [56], it was shown that the contributions to electric dipole moment of the electron and charged lepton flavour violating processes are much suppressed beyond the current bounds if new heavy vectorlike leptons have masses beyond $\sim 100 \mathrm{TeV}$. In our scenario, besides having $M_{\Psi} \gg 100 \mathrm{TeV}$, the constraints on the model parameters from the requirement of the longevity of $Y$ render these phenomenological constraints completely irrelevant.

\subsection{Does the Long-Lived Particle constitute most of the DM?}

Here we would like to study the abundance of the LLP in our model. The abundance of the LLP $Y$ is bounded from above by the DM abundance of the Universe. Clearly the abundance of a PeV LLP depends on the re-heating temperature $T_{\mathrm{RH}}$ of the Universe after inflation. If $T_{\mathrm{RH}} \ll M_{Y}$, no $Y$ would be generated. If $T_{\mathrm{RH}} \sim M_{Y}$, some amount of $Y$ would be generated. If $T_{\mathrm{RH}} \gg M_{Y}$, it is possible to generate significant amount of $Y$. Here we 
will consider the last and most constraining scenario and determine the constraints on our model parameters in order not to over-produce $Y$. First let us estimate what is the upper bound on a PeV Y abundance. From Planck 2013 measurement, the ratio between DM over baryon densities are [45]

$$
\frac{\Omega_{\mathrm{DM}}}{\Omega_{B}}=\frac{Y_{\mathrm{DM}} M_{\mathrm{DM}}}{Y_{B} M_{B}}=5.3,
$$

where we denote $Y_{x} \equiv n_{x} / s$, the $x$ number density normalized by the entropy density $s=\left(2 \pi^{2} / 45\right) g_{\star} T^{3}$. During the radiation dominated period of the Universe, the relativistic degrees of freedom $g_{\star}=106.75$, assuming only the SM particles. Taking the baryon mass $M_{B}=1 \mathrm{GeV}$ and $Y_{B}=8.8 \times 10^{-11}$, we have

$$
Y_{\mathrm{DM}}=1.2 \times 10^{-16}\left(\frac{4 \times 10^{6} \mathrm{GeV}}{M_{\mathrm{DM}}}\right) .
$$

Hence the above is the upper bound for the abundance $Y_{Y}$ of our LLP $Y$ of mass $M_{Y}$.

First, let us discuss the possibility to have the correct $Y_{Y}$ in the thermal freeze-out scenario. In this scenario, the scattering processes $Y Y \leftrightarrow H H, Y Y \leftrightarrow \ell_{L} \ell_{L}$ and $Y Y \leftrightarrow X_{I} X_{I}$ (the last scattering process is relevant only for global $\mathrm{U}(1)_{X}$ scenario) as shown in figure 9 are fast to keep $Y$ in thermal equilibrium at $T \gg M_{Y}$. Since the EW symmetry breaking remains unbroken, $\ell_{L}$ and $H$ refer to the SM lepton and Higgs doublets respectively. For gauged $\mathrm{U}(1)_{X}$, if $T_{\mathrm{RH}} \gg M_{g}$ with $M_{g}$ the $\mathrm{U}(1)_{X}$ gauge boson mass, this gauge interactions can also keep $Y$ in thermal equilibrium. The final $Y_{Y}$ can be estimated from the time when these scattering processes freeze out. In the following we will define the temperature as $z \equiv M_{Y} / T$. Due to fast reactions which keep $Y$ in equilibrium, $Y_{Y}$ is equal to its equilibrium abundance $Y_{Y}^{\mathrm{eq}}(z)=45 /\left(2 \pi^{4} g_{\star}\right) z^{2} \mathcal{K}_{2}(z)$ with $\mathcal{K}_{2}(z)$ the modified Bessel function of type 2 until the time of freeze-out at $z_{f o} \equiv M_{Y} / T_{f o}$. Hence we can estimate the final $Y_{Y} \approx Y_{Y}^{\mathrm{eq}}\left(z_{f o}\right) \approx 10^{-16}$ which gives us $z_{f o} \approx 35$. Such a late freeze-out implies large annihilating cross sections. We found that for $M_{Y} \sim \mathrm{PeV}$, such large cross sections cannot be obtained if $\lambda_{H Y}$ and $y_{\Psi}$ (also $\lambda_{X Y}$ for global $\mathrm{U}(1)_{X}$ case) are bounded to be perturbative i.e. $<4 \pi$. Since thermal freeze-out is not a viable scenario, for gauged $\mathrm{U}(1)_{X}$, we have to ensure that the gauge interactions between $Y$ and $\mathrm{U}(1)_{X}$ gauge boson are not in thermal equilibrium. Since the gauge coupling is generically large, we can only suppress this interaction by assuming $T_{\mathrm{RH}}$ much smaller than the mass of the gauge boson $M_{g}$ such that there is no gauge boson for gauge scatterings to take place.

In the following, we will study the scenario in which $Y$ abundance is obtained from the freeze-in scenario where $Y$ is very weakly coupled to other particles in the thermal bath and is generated from the interactions with those particles [57]. With the assumption $T_{\mathrm{RH}} \ll M_{g}$, the processes we have to consider are shown in figure 9 . We only show t-channel scattering processes and it is understood that in the calculation, one should also include the u-channel scattering processes. For gauged $\mathrm{U}(1)_{X}$, we have to consider processes in the top two rows of figure 9: $H H \leftrightarrow Y Y, \bar{\ell}_{L} \ell_{L} \leftrightarrow Y Y, \Psi_{R} \leftrightarrow \ell_{L} Y, H H \leftrightarrow X_{R} X_{R}$ and $X_{R} \leftrightarrow Y Y$. For global $\mathrm{U}(1)_{X}$, we also have to take into account the scattering with the NGB and consider two additional scatterings $H H \leftrightarrow X_{I} X_{I}$ and $X_{I} X_{I} \leftrightarrow Y Y$. 

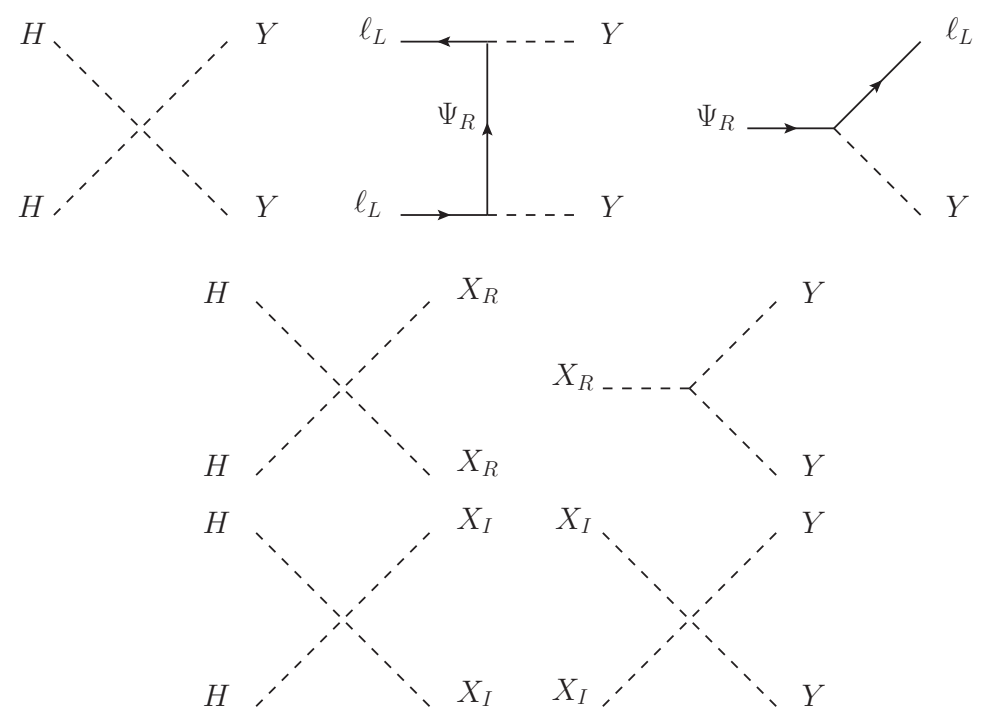

Figure 9. The scattering and decay processes which help to populate $Y$ abundance in the early Universe. For gauged $\mathrm{U}(1)_{X}$, only the processes in the top two rows are relevant while for global $\mathrm{U}(1)_{X}$, we also have to include the scattering processes with NGB in the last row. For the t-channel scattering processes, it is understood that we also have to include the u-channel scattering processes.

The SM particles $H$ and $\ell_{L}$ are necessarily in thermal equilibrium due to fast gauge interactions. If $T_{\mathrm{RH}} \gg M_{\Psi}$, the vectorlike fermion doublets $\Psi_{L}, \Psi_{R}$ which are charged under $\mathrm{SU}(2)_{L} \times \mathrm{U}(1)_{Y}$ will also be in thermal equilibrium due to fast gauge interactions. If $T_{\mathrm{RH}} \gg M_{X_{R}}$, taking the value allowed by invisible Higgs decay constraint (6.7) $\lambda_{H X}=$ $10^{-2}, X_{R}$ will also be thermal equilibrium due to the fast scatterings $H H \leftrightarrow X_{R} X_{R}$. Finally for the case of global $\mathrm{U}(1)_{X}$, taking $\lambda_{H X}=10^{-2}, X_{I}$ will also be in thermal equilibrium. On the other hand, $Y$ will be populated due to the scatterings $H, \ell_{L}$ and $X_{I}$ and the decays of $\Psi_{R}$ and $X_{R}$ as shown in figure 9 . We can write down the Boltzmann equation to describe the generation of $Y$

$$
\begin{aligned}
s \mathcal{H} z \frac{d Y_{Y}}{d z}= & 2\left(\gamma_{H H \leftrightarrow Y Y}+\gamma_{\ell_{L} \ell_{L} \leftrightarrow Y Y}\right)\left[1-\left(\frac{Y_{Y}}{Y_{Y}^{\mathrm{eq}}}\right)^{2}\right]+\gamma_{\Psi_{R} \leftrightarrow \ell_{L} Y}\left[1-\frac{Y_{Y}}{Y_{Y}^{\mathrm{eq}}}\right] \\
& +2\left(\gamma_{X_{R} \leftrightarrow Y Y}+\gamma_{X_{I} X_{I} \leftrightarrow Y Y}\right)\left[1-\left(\frac{Y_{Y}}{Y_{Y}^{\mathrm{eq}}}\right)^{2}\right],
\end{aligned}
$$

where $\gamma_{a}$ is the thermal averaged reaction density for the corresponding process $a$ and $\mathcal{H}=\sqrt{4 \pi^{3} / 45} \sqrt{g_{\star}} T^{2} / M_{\mathrm{Pl}}$ is the Hubble expansion rate with the Planck mass $M_{\mathrm{Pl}}=$ $1.22 \times 10^{19} \mathrm{GeV}$. In writing the equation above, we have also taken $H, \ell_{L}, \Psi_{R}, X_{R}$ and $X_{I}$ to be in thermal equilibrium. In fact, since $y_{\Psi} \ll 1$ (weakly coupled) and $\gamma_{\ell_{L} \ell_{L} \leftrightarrow Y Y} \propto y_{\Psi}^{4}$ while $\gamma_{\Psi_{R} \leftrightarrow \ell_{L} Y} \propto y_{\Psi}^{2}$, we will ignore the sub-dominant scattering process $\ell_{L} \ell_{L} \leftrightarrow Y Y$. In addition with $Y_{Y} \ll Y_{Y}^{\text {eq }}$, we can further simplify eq. (6.26) by dropping the terms with $Y_{Y} / Y_{Y}^{\mathrm{eq}}$. Finally we obtain a very simple Boltzmann equation

$$
s \mathcal{H} z \frac{d Y_{Y}}{d z}=2 \gamma_{H H \leftrightarrow Y Y}+\gamma_{\Psi_{R} \leftrightarrow \ell_{L} Y}+2 \gamma_{X_{R} \leftrightarrow Y Y}+2 \gamma_{X_{I} X_{I} \leftrightarrow Y Y}
$$


In the above the thermal averaged reaction densities are given by

$$
\begin{aligned}
\gamma_{H H \leftrightarrow Y Y} & =\frac{\lambda_{H Y}^{2}}{256 \pi^{5}} M_{Y}^{4} \frac{\left[\mathcal{K}_{1}(z)\right]^{2}}{z^{2}}, \\
\gamma_{\Psi_{R} \leftrightarrow \ell_{L} Y} & =\frac{\left|y_{\Psi}\right|^{2}}{32 \pi^{3}} a_{\Psi}^{3} M_{Y}^{4} \frac{\mathcal{K}_{1}\left(a_{\Psi} z\right)}{z}\left(1-\frac{1}{a_{\Psi}^{2}}\right)^{2}, \\
\gamma_{X_{R} \leftrightarrow Y Y} & =\frac{\lambda_{X Y}^{2}}{64 \pi^{3}} a_{X_{R}} w^{2} M_{Y}^{2} \frac{\mathcal{K}_{1}\left(a_{X_{R}} z\right)}{z} \sqrt{1-\frac{4}{a_{X_{R}}^{2}}}, \\
\gamma_{X_{I} X_{I} \leftrightarrow Y Y} & =\frac{\lambda_{X Y}^{2}}{2048 \pi^{5}} M_{Y}^{4} \frac{\left[\mathcal{K}_{1}(z)\right]^{2}}{z^{2}},
\end{aligned}
$$

where $a_{i} \equiv M_{i} / M_{Y}$ and we have summed over lepton flavour $\left|y_{\Psi}\right|^{2} \equiv \sum_{\alpha}\left|\left(y_{\Psi}\right)_{\alpha}\right|^{2}$. We can analytically solve eq. (6.27) and obtain the final $Y$ abundance as follows

$$
\begin{aligned}
Y_{Y} \simeq & 10^{-16}\left(\frac{106.75}{g_{\star}}\right)^{3 / 2}\left(\frac{4 \times 10^{6} \mathrm{GeV}}{M_{Y}}\right)\left[\left(\frac{\lambda_{H Y}}{4 \times 10^{-11}}\right)^{2}+\left(\frac{\left|y_{\Psi}\right|}{8 \times 10^{-12}}\right)^{2}\left(\frac{10}{a_{\Psi}}\right)\right. \\
& \left.+\left(\frac{\lambda_{X Y}}{1 \times 10^{-10}}\right)^{2}\left(\frac{10^{3}}{a_{X_{R}}}\right)^{3}\left(\frac{4 \times 10^{6} \mathrm{GeV}}{M_{Y}}\right)^{2}\left(\frac{w^{2}}{10^{10} \mathrm{GeV}}\right)^{2}+\left(\frac{\lambda_{X Y}}{1 \times 10^{-10}}\right)^{2}\right]
\end{aligned}
$$

In the solution above we have ignored the phase space factors in the decays which are negligible when $a_{\Psi}, a_{X_{R}} \gg 1$. In eq. (6.32), the contributions in the square bracket are in the following order $H H \leftrightarrow Y Y, \Psi_{R} \leftrightarrow \ell_{L} Y, X_{R} \leftrightarrow Y Y$ and $X_{I} X_{I} \leftrightarrow Y Y$. The last contribution is only relevant for the global $\mathrm{U}(1)_{X}$ scenario. We have verified the solution above by numerically solving eq. (6.26). All in all, we require $\left|y_{\Psi}\right|, \lambda_{X Y}, \lambda_{H Y} \lesssim 10^{-11}-10^{-10}$ in order not to have $Y$ exceeding the $\mathrm{PeV} \mathrm{DM}$ abundance $Y_{\mathrm{DM}} \sim 10^{-16}$ as in eq. (6.25).

Finally we would like to reiterate that in the fit of sections 4 and 5 , we have assumed the LLP constitutes all the DM. If the LLP constitutes a fraction $\kappa$ of the DM, then its lifetime has to be shorter by a factor of $\kappa$ in order to maintain the observed flux by IceCube. Hence, we cannot choose an arbitrarily small $\kappa$ and at some point when $\kappa \tau_{Y}<t_{0}$, it will cease to be the LLP. Since $\tau_{Y} \propto \mu_{X Y}^{-2}$ (see eqs. (6.10)-(6.12)), to obtain $\kappa \tau_{Y}, \mu_{X Y}$ has to increase by factor of $1 / \sqrt{\kappa}$. In the gauged $\mathrm{U}(1)_{X}$ scenario, we are allowed to take $\kappa$ as low as $\sim 10^{-10}$. On the other hand, for the global $\mathrm{U}(1)_{X}$ scenario, due to the fast decay of $Y$ into NGB, we require $\kappa \gtrsim 10^{-4}$ which can be inferred from eqs. (6.13) and (6.14). In other words, the LLP has to constitute at least about $0.01 \%$ of the DM in the global U(1) $X$ scenario.

\section{Conclusion}

The serendipitous discovery of high-energy extraterrestrial neutrinos by IceCube inaugurates an extremely exciting era for neutrino astronomy. It is clear that the neutrino candidate events above $60 \mathrm{TeV}$ cannot be explained by atmospheric neutrinos alone. Moreover, the absence of events in the region $500 \mathrm{TeV}-1 \mathrm{PeV}$ and above $2 \mathrm{PeV}$ seem to suggest a neutrino spectrum beyond a single power-law. This will have to be confirmed or rejected by future data. 
We investigate the possibility that the IceCube neutrino events with energies from $30 \mathrm{TeV}$ to $2 \mathrm{PeV}$ can be explained by (a) solely a power-law spectrum, (b) decays of a PeV scale LLP with power-law spectrum component and (c) solely decays of a PeV scale LLP. For scenario (c), we study a simple scenario where a scalar LLP, $Y$, decays dominantly into $Y \rightarrow \nu N, Y \rightarrow 2 h$ or $Y \rightarrow 4 h$. We present a simple extension of the SM where two extra complex scalars, singlets under the SM group, a vectorlike pair of fermion doublets and a right-handed fermion singlet are charged under a global or gauged $\mathrm{U}(1)_{X}$. We show that this model can give rise to a LLP that can decay dominantly to the above modes while the LLP abundance is generated through the freeze-in mechanism. In particular, we find that if $\mathrm{U}(1)_{X}$ is a global symmetry, such LLP has to constitute at least $0.01 \%$ of the total DM of the Universe due to the long-lived constraint.

Using the current IceCube three-year data set of 37 events [38], we find that all the three scenarios (a)-(c) above fit the data equally well due to the low statistics. Our results for these fits are summarized in table 1. In order to disentangle various scenarios above, we simulate the future IceCube data based on current hypotheses up to neutrino energies of $10 \mathrm{PeV}$. Using only the energy spectrum information, we determine the exposure time needed to disentangle various scenarios by future data (summarized in table 2). Assuming a power-law spectrum, the solely LLP decay models can be excluded with two to five times the current data. Other combinations will take longer time and in some cases it will be impossible to distinguish between hypotheses.

\section{Acknowledgments}

This work was supported by Fundação de Amparo à Pesquisa do Estado de São Paulo (FAPESP) and Conselho Nacional de Ciência e Tecnologia (CNPq). H.M. thanks Universidade de São Paulo for the great opportunity of stay under "Programa de Bolsas para Professors Visitantes Internacionais na USP". H.M. and R.Z.F. thank the Kavli Institute for Theoretical Physics in UC Santa Barbara for its hospitality, where part of this work was completed: this research was supported in part by the National Science Foundation under Grant No. NSF PHY11-25915. We also acknowledge partial support from the European Union FP7 ITN INVISIBLES (Marie Curie Actions, PITN-GA-2011-289442). We are grateful to Shigeru Yoshida for informative correspondences, and H.M. and R.Z.F. thank Kohta Murase for discussions. C.S.F. thanks Jordi Salvado for helpful discussions.

\section{A Some details on the statistical treatment}

For the statistical treatment we mainly follow [58, 59]. Through this work we consider several hypotheses in order to explain the data observed by IceCube. In this appendix we refer to any of these hypotheses as $\mathrm{H}_{0}\left(\theta_{i}\right)$, where $\theta_{i}$ are the free parameters of the hypotheses. In order to estimate the preferred values of $\theta_{i}$, we maximize the likelihood function

$$
L\left(\theta_{i} ; k_{n}\right)=\prod_{n=1}^{12} f\left(k_{n} ; \mu_{n}\left(\theta_{i}\right)\right)
$$


with respect to $\theta_{i}$, where $f\left(k_{n} ; \mu_{n}\left(\theta_{i}\right)\right)$ is the Poisson probability distribution function for measuring $k_{n}$ events in the bin $n$ assuming that the mean value is given by $\mu_{n}\left(\theta_{i}\right)$. Notice that the results obtained from this procedure are equivalent to those obtained from the minimization of the function $\chi^{2}\left(\theta_{i} ; k_{n}\right)=-2 \ln \left(L\left(\theta_{i} ; k_{n}\right)\right)$.

Therefore, for simplicity and numerical stability, we normally use $\chi^{2}\left(\theta_{i} ; k_{n}\right)$ to report our results. The estimators of the parameters $\theta_{i}$ are defined as $\hat{\theta}_{i}$, the minimum of the statistic is given by $\chi_{\min }^{2} \equiv \chi^{2}\left(\hat{\theta}_{i}, k_{n}\right)$ and the mean values of the hypothesis $\mathrm{H}_{0}\left(\hat{\theta}_{i}\right)$ are given by $\mu_{n}\left(\hat{\theta}_{i}\right)$. In the following we show how we compute the p-value associated to the observed $\chi_{\min }^{2}$ and the corresponding confidence intervals for the variables $\theta_{i}$.

\section{A.1 p-value}

In order to quantify the level of agreement or incompatibility of the hypothesis $\mathrm{H}_{0}\left(\hat{\theta}_{i}\right)$ with respect to the current data, it is useful to evaluate the probability of obtaining the current value of $\chi_{\min }^{2}$ assuming that the data is indeed generated by the hypothesis $\mathrm{H}_{0}\left(\hat{\theta}_{i}\right)$.

In practice, we must construct the probability distribution function, $f\left(t \mid \mathrm{H}_{0}\right)$, with $t=\chi_{\min }^{2}\left(\theta_{i}, \bar{k}_{n}\right)$ and $\bar{k}_{n} \sim \operatorname{Pois}\left(\mu_{n}(\hat{\theta})\right)$. In general, this function is given by the normalized frequency distribution of $t$ obtained from several random realizations of $\mathrm{H}_{0}(\hat{\theta})$. Using $f\left(t \mid \mathrm{H}_{0}\right)$ we are able to compute the p-value associated to $\chi_{\min }^{2}$, which is defined as the probability to find $t$ in the region of lesser or equal incompatibility with $\mathrm{H}_{0}$ than the level of incompatibility observed with the current data,

$$
p=\int_{\chi_{\min }^{2}}^{\infty} f\left(t \mid \mathrm{H}_{0}\right) d t
$$

Thus, a very small value of $p$ implies that the observed level of incompatibility is quite unlikely to be found, which also suggest that $\mathrm{H}_{0}$ is not a good representation of the data. When this is the case, it is said that the hypothesis $\mathrm{H}_{0}$ is rejected at $(1-p)$ confidence level.

\section{A.2 Intervals}

For simplicity, we compute the intervals using a Bayesian point of view. From Bayes theorem, the probability density function of the parameters $\theta_{i}$ can be obtained from the product of the likelihood function $L\left(\theta_{i} ; k_{n}\right)$ and the joint prior of the parameters $\theta_{i}$, which we define as $\pi\left(\theta_{i}\right)$. We assume that each prior is constant in a finite interval and null otherwise. Thus, the domain of the variables $\theta_{i}$ is given by a finite hyper volume $U$, which is obtained from the product of the priors. The normalized p.d.f. for $\theta_{i} \in U$ is given by,

$$
p\left(\theta_{i}\right)=\frac{L\left(\theta_{i} ; k_{n}\right)}{\int_{U} L\left(\bar{\theta}_{i} ; k_{n}\right) d \bar{\theta}_{i}} .
$$

From the above expression, it is direct to compute the coverage probability of a region of parameters $V \in U$, since we just have to integrate the previous expression on the region $V$. However, it is not direct to obtain an interval such that the coverage probability is some fixed number $(1-\alpha)$, because this procedure involves an integral equation. Furthermore, it is not easy to find a rule to discriminate within degenerate solutions. These problems can be addressed in a simple way when we consider the discrete form of eq. (A.3). 
Therefore, we generate a quite big sample of points $\theta_{i}$ covering the domain $U$. In general we use $N=\left(10^{4}, 500^{2}, 200^{3}\right)$ when the number of free parameters is $n=(1,2,3)$ respectively. In the same procedure we are able to compute the pairs $\left\{\theta_{i}, L\left(\theta_{i} ; k_{n}\right)\right\}_{j}$, with $j=1 \ldots N$. Using the elements of this set we can compute the coverage probability of some particular region, but first we need to fix the rule to discriminate within degenerate intervals. For instance, we require that the resulting interval contains the most likely points. This is analogous to considering a symmetric interval around the center of a Gaussian distributed variable. To implement this requirement, we just order the set $\left\{\theta_{i}, L\left(\theta_{i} ; k_{n}\right)\right\}_{j}$ in descending order in $L\left(\theta_{i} ; k_{n}\right)$ such that the new set satisfies the condition $L\left(\theta_{i} ; k_{n}\right)_{j} \geq$ $L\left(\theta_{i} ; k_{n}\right)_{k}$ with $k=j+1 \ldots N$. Finally, the hyper volume with $(1-\alpha)$ coverage probability is given by the set $\left\{\theta_{i}, L\left(\theta_{i} ; k_{n}\right)\right\}_{j}$ with $j=1 \ldots M$, such that

$$
\frac{\sum_{j=1}^{M} L\left(\theta_{i} ; k_{n}\right)_{j}}{\sum_{l=1}^{N} L\left(\theta_{i} ; k_{n}\right)_{l}} \leq(1-\alpha),
$$

where we have assumed that the volume element $\Delta \theta_{i}$ is constant everywhere. The final procedure only involves a loop on the variable $j$ that runs in unit steps until the condition (A.4) is violated, which determines the value of $M$. The intervals at some confidence level for a given variable $\theta_{i}$ correspond to the extreme values of the set $\left\{\theta_{i}, L\left(\theta_{i} ; k_{n}\right)\right\}_{j}$ with $j=1 \ldots M$.

\section{A.3 Exclusion time}

In this section we compute the necessary exposure time in order to exclude a given hypothesis $\mathrm{H}_{\mathrm{j}}$ assuming that the hypothesis $\mathrm{H}_{\mathrm{i}}$ is the true explanation of the current data. This procedure is based in the computation of p-values between pairs of hypotheses at different times. Below, we describe the algorithm step by step:

1. In general, we compute the mean values of some hypothesis $\mathrm{H}_{0}\left(\theta_{i}\right)$ at a time $T=$ $f_{T} \times 988$ days from the product of the mean values predicted today, defined as $\mu_{n}\left(\theta_{i}\right)$, times the factor $f_{T}$. Thus, $\mu_{n}\left(\hat{\theta}_{i}, T\right)=\mu_{n}\left(\hat{\theta}_{i}\right) \times f_{T}$. For simplicity we call the version of $\mathrm{H}_{0}\left(\theta_{i}\right)$ at a time $T$ as $\mathrm{H}_{0}\left(\theta_{i}, T\right)$.

2. Assuming that the hypothesis $\mathrm{H}_{\mathrm{i}}\left(\hat{\theta}_{i}, T\right)$ is the true hypothesis, we compute the number of expected pseudo events $\bar{k}_{n}(T)$ at a fixed time $T$. As these numbers follow a random distribution, such that $\bar{k}_{n}(T) \sim \operatorname{Pois}\left(\mu_{n}\left(\hat{\theta}_{i}, T\right)\right)$, we notice that there is not a unique way to determine their values. Then, in order to define a stable algorithm we just pick the most likely values of $\bar{k}_{n}(T)$ given $\mu_{n}\left(\hat{\theta}_{i}, T\right)$. This approach covers the most likely outputs of an hypothetical experiment and it is stable under repetitions of the procedure.

3. We take the hypothesis $\mathrm{H}_{\mathrm{j}}\left(\theta_{i}, T\right)$ with $f_{T}$ fixed and $\theta_{i}$ free. We estimate the best fit parameters of the hypothesis $\mathrm{H}_{\mathrm{j}}\left(\theta_{i}, T\right)$ using the likelihood method with respect to the observed pseudo events generated from $\mathrm{H}_{\mathrm{i}}\left(\hat{\theta}_{i}, T\right)$. Finally, we compute the p-value of this fit. We choose a minimum p-value such that both hypotheses are incompatible. Following a conservative approach we consider $p=0.05$ as the threshold. 


\section{B Confidence intervals}

The summary of the confidence intervals for each hypothesis $\mathrm{H}_{0}$ are showed in table 4 .

\begin{tabular}{|c|c|c|c|c|c|}
\hline $\mathrm{H}_{0}$ & Best fit & $U$ (prior) & $\pm 1 \sigma(68 \%)$ & $\pm 2 \sigma(95 \%)$ & $\pm 3 \sigma(99 \%)$ \\
\hline \multirow{4}{*}{ I } & \multirow{2}{*}{$s=2.3$} & \multirow{2}{*}[0,4]{} & 2.83 & 3.21 & 3.46 \\
\hline & & & 1.72 & 1.12 & 0.57 \\
\hline & \multirow{2}{*}{$C_{0}=0.6$} & \multirow{2}{*}[10^{-4},10]{$^{*}$} & 1.41 & 2.08 & 2.51 \\
\hline & & & 0.12 & 0.01 & $1.33 \times 10^{-3}$ \\
\hline \multirow{6}{*}{ II.a } & \multirow{2}{*}{$s=2.43$} & \multirow{2}{*}[0,4]{} & 3.5 & 4.0 & 4.0 \\
\hline & & & 0.38 & 0.0 & 0.0 \\
\hline & \multirow{2}{*}{$C_{0}=0.51$} & \multirow{2}{*}[10^{-4},10]{$^{*}$} & 1.66 & 2.1 & 2.35 \\
\hline & & & $2.25 \times 10^{-3}$ & $10^{-4}$ & $10^{-4}$ \\
\hline & \multirow{2}{*}{$\tau_{Y}=5.26$} & \multirow{2}{*}[0.01,100]{$^{*}$} & 100 & 100 & 100 \\
\hline & & & 1.17 & 0.85 & 0.74 \\
\hline \multirow{6}{*}{ II.b } & \multirow{2}{*}{$s=2.76$} & \multirow{2}{*}[0,4]{} & 4.0 & 4.0 & 4.0 \\
\hline & & & 0.24 & 0.0 & 0.0 \\
\hline & \multirow{2}{*}{$C_{0}=0.52$} & \multirow{2}{*}[10^{-4},10]{$^{*}$} & 1.4 & 1.88 & 2.22 \\
\hline & & & $10^{-4}$ & $10^{-4}$ & $10^{-4}$ \\
\hline & \multirow{2}{*}{$\tau_{Y}=2.72$} & \multirow{2}{*}[0.01,100]{$^{*}$} & 100 & 100 & 100 \\
\hline & & & 1.02 & 0.74 & 0.62 \\
\hline \multirow{4}{*}{ III.b } & \multirow{2}{*}{$r_{\nu N}=0.35$} & \multirow{2}{*}[0,1]{} & 0.99 & 1.0 & 1.0 \\
\hline & & & 0.1 & 0.03 & 0.01 \\
\hline & \multirow{2}{*}{$\tau_{Y}=0.88$} & \multirow{2}{*}[0.01,100]{$^{*}$} & 2.41 & 6.65 & 13.09 \\
\hline & & & 0.48 & 0.36 & 0.31 \\
\hline \multirow{4}{*}{ IV.b } & \multirow{2}{*}{$r_{\nu N}=0.23$} & \multirow{2}{*}[0,1]{} & 0.91 & 1.0 & 1.0 \\
\hline & & & 0.0 & 0.0 & 0.0 \\
\hline & \multirow{2}{*}{$\tau_{Y}=1.13$} & \multirow{2}{*}[0.01,100]{$^{*}$} & 2.64 & 5.94 & 11.54 \\
\hline & & & 0.64 & 0.48 & 0.41 \\
\hline \multirow{2}{*}{$\mathrm{V}$} & \multirow{2}{*}{$\tau_{Y}=1.9$} & \multirow{2}{*}[0.01,100]{$^{*}$} & 3.67 & 8.71 & 16.56 \\
\hline & & & 1.13 & 0.74 & 0.6 \\
\hline
\end{tabular}

Table 4. Confidence level intervals. The lifetime $\tau_{Y}$ is in units of $\times 10^{28} \mathrm{~s}$, the normalization of the power-law $C_{0}$ is in units of $\mathrm{GeV} \mathrm{cm}{ }^{-2} \mathrm{sr}^{-1} \mathrm{~s}^{-1}$ and $r_{\nu N}$ is the branching ratio of the channel $Y \rightarrow \nu N$. The intervals with format $[a, b]^{*}$ are scanned in logarithmic scale. 
Open Access. This article is distributed under the terms of the Creative Commons Attribution License (CC-BY 4.0), which permits any use, distribution and reproduction in any medium, provided the original author(s) and source are credited.

\section{References}

[1] SNO collaboration, S.N. Ahmed et al., Measurement of the total active B-8 solar neutrino flux at the Sudbury Neutrino Observatory with enhanced neutral current sensitivity, Phys. Rev. Lett. 92 (2004) 181301 [nucl-ex/0309004] [INSPIRE].

[2] Super-Kamiokande collaboration, Y. Fukuda et al., Evidence for oscillation of atmospheric neutrinos, Phys. Rev. Lett. 81 (1998) 1562 [hep-ex/9807003] [INSPIRE].

[3] Super-Kamiokande collaboration, Y. Fukuda et al., Study of the atmospheric neutrino flux in the multi-GeV energy range, Phys. Lett. B 436 (1998) 33 [hep-ex/9805006] [INSPIRE].

[4] KamLAND collaboration, K. Eguchi et al., First results from KamLAND: Evidence for reactor anti-neutrino disappearance, Phys. Rev. Lett. 90 (2003) 021802 [hep-ex/0212021] [INSPIRE].

[5] DAYA-BAY collaboration, F.P. An et al., Observation of electron-antineutrino disappearance at Daya Bay, Phys. Rev. Lett. 108 (2012) 171803 [arXiv:1203.1669] [INSPIRE].

[6] MINOS collaboration, D.G. Michael et al., Observation of muon neutrino disappearance with the MINOS detectors and the NuMI neutrino beam, Phys. Rev. Lett. 97 (2006) 191801 [hep-ex/0607088] [INSPIRE].

[7] T2K collaboration, K. Abe et al., Indication of Electron Neutrino Appearance from an Accelerator-produced Off-axis Muon Neutrino Beam, Phys. Rev. Lett. 107 (2011) 041801 [arXiv: 1106.2822] [INSPIRE].

[8] ATLAS collaboration, A particle consistent with the Higgs Boson observed with the ATLAS Detector at the Large Hadron Collider, Science 338 (2012) 1576 [INSPIRE].

[9] CMS collaboration, A New Boson with a Mass of $125 \mathrm{GeV}$ Observed with the CMS Experiment at the Large Hadron Collider, Science 338 (2012) 1569 [inSPIRE].

[10] ICECuBE collaboration, M.G. Aartsen et al., Evidence for High-Energy Extraterrestrial Neutrinos at the IceCube Detector, Science 342 (2013) 1242856 [arXiv:1311.5238] [INSPIRE].

[11] E. Roulet, G. Sigl, A. van Vliet and S. Mollerach, PeV neutrinos from the propagation of ultra-high energy cosmic rays, JCAP 01 (2013) 028 [arXiv:1209.4033] [INSPIRE].

[12] I. Cholis and D. Hooper, On The Origin of IceCube's PeV Neutrinos, JCAP 06 (2013) 030 [arXiv:1211.1974] [INSPIRE].

[13] O.E. Kalashev, A. Kusenko and W. Essey, PeV neutrinos from intergalactic interactions of cosmic rays emitted by active galactic nuclei, Phys. Rev. Lett. 111 (2013) 041103 [arXiv: 1303.0300] [INSPIRE].

[14] F.W. Stecker, PeV neutrinos observed by IceCube from cores of active galactic nuclei, Phys. Rev. D 88 (2013) 047301 [arXiv: 1305.7404] [INSPIRE].

[15] K. Murase, M. Ahlers and B.C. Lacki, Testing the Hadronuclear Origin of PeV Neutrinos Observed with IceCube, Phys. Rev. D 88 (2013) 121301 [arXiv:1306.3417] [InSPIRE]. 
[16] L.A. Anchordoqui, H. Goldberg, M.H. Lynch, A.V. Olinto, T.C. Paul and T.J. Weiler, Pinning down the cosmic ray source mechanism with new IceCube data, Phys. Rev. D 89 (2014) 083003 [arXiv: 1306.5021] [INSPIRE].

[17] K. Murase and K. Ioka, TeV-PeV Neutrinos from Low-Power Gamma-Ray Burst Jets inside Stars, Phys. Rev. Lett. 111 (2013) 121102 [arXiv:1306.2274] [INSPIRE].

[18] W. Winter, Photohadronic Origin of the TeV-PeV Neutrinos Observed in IceCube, Phys. Rev. D 88 (2013) 083007 [arXiv: 1307.2793] [INSPIRE].

[19] M. Ahlers and K. Murase, Probing the Galactic Origin of the IceCube Excess with Gamma-Rays, Phys. Rev. D 90 (2014) 023010 [arXiv:1309.4077] [INSPIRE].

[20] A.M. Taylor, S. Gabici and F. Aharonian, Galactic halo origin of the neutrinos detected by IceCube, Phys. Rev. D 89 (2014) 103003 [arXiv:1403.3206] [INSPIRE].

[21] S. Sahu and L.S. Miranda, Some possible sources of IceCube TeV-PeV neutrino events, arXiv:1408.3664 [INSPIRE].

[22] B. Feldstein, A. Kusenko, S. Matsumoto and T.T. Yanagida, Neutrinos at IceCube from Heavy Decaying Dark Matter, Phys. Rev. D 88 (2013) 015004 [arXiv:1303.7320] [InSPIRE].

[23] Y. Bai, R. Lu and J. Salvado, Geometric Compatibility of IceCube TeV-PeV Neutrino Excess and its Galactic Dark Matter Origin, arXiv:1311.5864 [INSPIRE].

[24] A. Esmaili and P.D. Serpico, Are IceCube neutrinos unveiling PeV-scale decaying dark matter?, JCAP 11 (2013) 054 [arXiv:1308.1105] [INSPIRE].

[25] A. Bhattacharya, M.H. Reno and I. Sarcevic, Reconciling neutrino flux from heavy dark matter decay and recent events at IceCube, JHEP 06 (2014) 110 [arXiv:1403.1862] [INSPIRE].

[26] A. Bhattacharya, R. Gandhi and A. Gupta, The Direct Detection of Boosted Dark Matter at High Energies and PeV events at IceCube, arXiv:1407.3280 [INSPIRE].

[27] A. Esmaili, S.K. Kang and P.D. Serpico, IceCube events and decaying dark matter: hints and constraints, JCAP 12 (2014) 054 [arXiv:1410.5979] [INSPIRE].

[28] V. Barger and W.-Y. Keung, Superheavy Particle Origin of IceCube PeV Neutrino Events, Phys. Lett. B 727 (2013) 190 [arXiv:1305.6907] [InSPIRE].

[29] S. Pakvasa, A. Joshipura and S. Mohanty, Explanation for the low flux of high energy astrophysical muon-neutrinos, Phys. Rev. Lett. 110 (2013) 171802 [arXiv:1209.5630] [INSPIRE].

[30] T. Higaki, R. Kitano and R. Sato, Neutrinoful Universe, JHEP 07 (2014) 044 [arXiv: 1405.0013] [INSPIRE].

[31] Y. Ema, R. Jinno and T. Moroi, Cosmic-Ray Neutrinos from the Decay of Long-Lived Particle and the Recent IceCube Result, Phys. Lett. B 733 (2014) 120 [arXiv:1312.3501] [INSPIRE].

[32] Y. Ema, R. Jinno and T. Moroi, Cosmological Implications of High-Energy Neutrino Emission from the Decay of Long-Lived Particle, JHEP 10 (2014) 150 [arXiv:1408.1745] [INSPIRE].

[33] K. Ioka and K. Murase, IceCube PeV-EeV neutrinos and secret interactions of neutrinos, PTEP 2014 (2014) 061E01 [arXiv: 1404.2279] [INSPIRE]. 
[34] K.C.Y. Ng and J.F. Beacom, Cosmic neutrino cascades from secret neutrino interactions, Phys. Rev. D 90 (2014) 065035 [arXiv: 1404.2288] [InSPIRE].

[35] J.F. Cherry, A. Friedland and I.M. Shoemaker, Neutrino Portal Dark Matter: From Dwarf Galaxies to IceCube, arXiv:1411.1071 [INSPIRE].

[36] C.-Y. Chen, P.S. Bhupal Dev and A. Soni, Standard model explanation of the ultrahigh energy neutrino events at IceCube, Phys. Rev. D 89 (2014) 033012 [arXiv: 1309.1764] [INSPIRE].

[37] C.-Y. Chen, P.S.B. Dev and A. Soni, A Possible Two-component Flux for the High Energy Neutrino Events at IceCube, arXiv:1411.5658 [INSPIRE].

[38] ICECube collaboration, M.G. Aartsen et al., Observation of High-Energy Astrophysical Neutrinos in Three Years of IceCube Data, Phys. Rev. Lett. 113 (2014) 101101 [arXiv: 1405.5303] [INSPIRE].

[39] ICECube collaboration, M.G. Aartsen et al., First observation of PeV-energy neutrinos with IceCube, Phys. Rev. Lett. 111 (2013) 021103 [arXiv: 1304.5356] [INSPIRE].

[40] M. Kachelriess, P.D. Serpico and M.A. Solberg, On the role of electroweak bremsstrahlung for indirect dark matter signatures, Phys. Rev. D 80 (2009) 123533 [arXiv:0911.0001] [INSPIRE].

[41] P. Ciafaloni, D. Comelli, A. Riotto, F. Sala, A. Strumia and A. Urbano, Weak Corrections are Relevant for Dark Matter Indirect Detection, JCAP 03 (2011) 019 [arXiv:1009.0224] [INSPIRE].

[42] L. Covi, M. Grefe, A. Ibarra and D. Tran, Neutrino Signals from Dark Matter Decay, JCAP 04 (2010) 017 [arXiv:0912.3521] [INSPIRE].

[43] T. Sjöstrand, S. Mrenna and P.Z. Skands, A Brief Introduction to PYTHIA 8.1, Comput. Phys. Commun. 178 (2008) 852 [arXiv:0710.3820] [INSPIRE].

[44] A. Ibarra and D. Tran, Gamma Ray Spectrum from Gravitino Dark Matter Decay, Phys. Rev. Lett. 100 (2008) 061301 [arXiv:0709.4593] [INSPIRE].

[45] Planck collaboration, P.A.R. Ade et al., Planck 2013 results. XVI. Cosmological parameters, Astron. Astrophys. 571 (2014) A16 [arXiv:1303.5076] [INSPIRE].

[46] C.-R. Chen, F. Takahashi and T.T. Yanagida, Gamma rays and positrons from a decaying hidden gauge boson, Phys. Lett. B 671 (2009) 71 [arXiv:0809.0792] [InSPIRE].

[47] A. Loeb and E. Waxman, The Cumulative background of high energy neutrinos from starburst galaxies, JCAP 05 (2006) 003 [astro-ph/0601695] [INSPIRE].

[48] ICECube collaboration, M.G. Aartsen et al., Atmospheric and astrophysical neutrinos above 1 TeV interacting in IceCube, Phys. Rev. D 91 (2015) 022001 [arXiv:1410.1749] [InSPIRE].

[49] K. Murase and J.F. Beacom, Constraining Very Heavy Dark Matter Using Diffuse Backgrounds of Neutrinos and Cascaded Gamma Rays, JCAP 10 (2012) 043 [arXiv: 1206.2595] [INSPIRE].

[50] The Fermi LAT collaboration, M. Ackermann et al., The spectrum of isotropic diffuse gamma-ray emission between $100 \mathrm{MeV}$ and $820 \mathrm{GeV}$, Astrophys. J. 799 (2015) 86 [arXiv: 1410.3696] [INSPIRE].

[51] K. Murase, private communications.

[52] M. Garny, A. Ibarra and D. Tran, Constraints on Hadronically Decaying Dark Matter, JCAP 08 (2012) 025 [arXiv: 1205.6783] [INSPIRE]. 
[53] D. Chang, P.B. Pal and G. Senjanović, Axions From Chiral Family Symmetry, Phys. Lett. B 153 (1985) 407 [INSPIRE].

[54] G. Bélanger, B. Dumont, U. Ellwanger, J.F. Gunion and S. Kraml, Status of invisible Higgs decays, Phys. Lett. B 723 (2013) 340 [arXiv:1302.5694] [InSPIRE].

[55] S. Weinberg, Baryon and Lepton Nonconserving Processes, Phys. Rev. Lett. 43 (1979) 1566 [INSPIRE].

[56] K. Ishiwata and M.B. Wise, Phenomenology of heavy vectorlike leptons, Phys. Rev. D 88 (2013) 055009 [arXiv: 1307.1112] [INSPIRE].

[57] L.J. Hall, K. Jedamzik, J. March-Russell and S.M. West, Freeze-In Production of FIMP Dark Matter, JHEP 03 (2010) 080 [arXiv:0911.1120] [INSPIRE].

[58] L. Lyons, Statistics For Nuclear And Particle Physicists, Cambridge University Press, Cambridge, U.K. (1986), pg. 226.

[59] Particle Data Group collaboration, K.A. Olive et al., Review of Particle Physics, Chin. Phys. C 38 (2014) 090001. 